\title{
Conductive-radiative heat transfer in a 2D semi-transparent medium with a square centered obstacle
}

\author{
Julien Sorel Djeumegni ${ }^{1} *$, Myriam Lazard ${ }^{1}$, Vital Le Dez ${ }^{1}$ \\ Hervé Thierry Tagne Kamdem ${ }^{2} \dagger$ \\ ${ }^{1}$ Institut Pprime, Université de Poitiers, CNRS, ENSMA UPR 3346, 2 Rue Pierre Brousse, \\ Bâtiment B25, TSA 41105,86073, Poitiers Cedex 9, France. \\ ${ }^{2}$ Unité de Recherche de Mécanique et de modélisation de Systèmes Physiques (UR2MSP), \\ Département de Physique/Faculté des Sciences, Université de Dschang, Cameroun. \\ † In memoriam (1973-2020) \\ *Corresponding author. E-mail: julien.sorel.djeumegni@univ-poitiers.fr
}

\begin{abstract}
The present paper deals with an exact semi-analytical formulation of a combined conductiveradiative heat transfer, applied to a two-dimensional semi-transparent medium carrying a square centered obstacle. The gray participating medium with black boundaries absorbs, emits but does not scatter radiation. One intends to evaluate the temperature and radiative heat flux distributions within the semi-transparent medium. The radiative transfer equation has been solved using an exact analytical expansion of Bickley-Naylor and Altaç angular integrated Bickley-Naylor functions, then solved numerically with Gauss quadrature. Energy equation has been directly discretized and approximated numerically using the centered finite differences method and consequently the dimensionless temperature has been obtained after an iterative scheme. The results of radiative quantities obtained have been verified with benchmark with an excellent agreement, both for simple and complex geometries. Simulations have been performed to obtain results for different sizes of the centered obstacle and the optical thickness. The effects of the conduction-radiation parameters, discrete directions and the size of the obstacle have also been investigated.
\end{abstract}

Keywords: conductive-radiative, semi-analytical, obstacle, semi-transparent medium

\section{Nomenclature}

$\begin{array}{cl}\text { Bis }_{n} & \text { Altaç angular integrated Bickley-Naylor functions } \\ C_{p} & \text { specific heat }\left(J K^{-1}\right) \\ C^{\Omega} & \text { unit disk of } \mathbb{R}^{2}: C^{\Omega}=\left\{\Omega \in \mathbb{R}^{2} /|\Omega| \leq 1\right\} \\ \left(\vec{e}_{x}, \vec{e}_{y}\right) & \text { unit vectors of } x, y \text { directions } \\ G & \text { volumic incident radiation }\left(W m^{-3}\right)\end{array}$




\begin{tabular}{|c|c|}
\hline$G^{*}$ & dimensionless incident radiation \\
\hline$H$ & length of external cavity along $x$ and $y$ direction $(m)$ \\
\hline$h$ & length of the inner square body $(\mathrm{m})$ \\
\hline$(i, j, p, q)$ & cells numbering \\
\hline I & one directional incoming radiation intensity $\left(\mathrm{Wm}^{-2} \mathrm{Sr}\right)$ \\
\hline$I_{0}$ & black body radiation intensity $\left(W m^{-2} S r\right)$ \\
\hline$k$ & time index \\
\hline$k_{a}$ & absorption coefficient $\left(m^{-1}\right)$ \\
\hline$k_{c}$ & thermal conductivity $\left(W m^{-1} K^{-1}\right)$ \\
\hline$K i_{n}$ & Bickley-Naylor functions \\
\hline$l, m$ & angular and spatial numbering quadrature \\
\hline$N_{\varphi}, N_{x}, N_{y}$ & number of angular quadrature, and cells \\
\hline$N_{t}$ & maximum number of time steps \\
\hline$N_{p l}$ & conduction-radiation parameter \\
\hline$\vec{q}^{r}$ & radiative flux vector $\left(W m^{-2}\right)$ \\
\hline$\vec{q}_{r}^{*}$ & dimensionless radiative flux vector $\left(\mathrm{Wm}^{-2}\right)$ \\
\hline$\vec{q}^{c}$ & conductive heat flux $\left(W^{-2}\right)$ \\
\hline$q_{x}$ & $x$-component of the conductive-radiative heat flux $\left(\mathrm{Wm}^{-2}\right)$ \\
\hline$q_{y}$ & $y$-component of the conductive-radiative heat flux $\left(\mathrm{Wm}^{-2}\right)$ \\
\hline$\vec{S}^{r}$ & radiative source vector $\left(W^{-3}\right)$ \\
\hline$S$ & curvilinear abscissa \\
\hline$t$ & time $(s)$ \\
\hline$T$ & radiation temperature $(K)$ \\
\hline$T_{0}$ & initial temperature in the medium \\
\hline$T_{\text {ref }}$ & reference temperature \\
\hline$T^{*}$ & dimensionless temperature in the medium \\
\hline$u$ & real number at which $B_{i s_{n}}$ is evaluated \\
\hline
\end{tabular}

Greek symbols

$\begin{array}{cl}\Delta x, \Delta y & \text { space step along } x \text { and } y \text { respectively }(m) \\ \Delta t & \text { time step } \\ \Delta \xi & \text { dimensionless step time } \\ \vec{\nabla}, \Delta & \text { Gradient and Laplacian operators respectively } \\ \beta_{l} & \text { angular abscissa } \\ \sigma_{B} & \text { Stefan-Boltzmann constant }\left(5.6710^{-8} \mathrm{Wm}^{-2} \mathrm{~K}^{-4}\right) \\ \varphi, \theta & \text { azimuthal and zenith angle of unit vector } \vec{\Omega} \\ \vec{\Omega} & \text { unit radiation propagation vector } \\ \nabla \cdot \vec{q}_{r} & \text { radiative flux divergence }\left(\mathrm{Wm}^{-3}\right) \\ \delta & \text { radiative path length from boundary surface to attenuated point in the } \\ \omega_{l}, \omega_{m} & \text { Medium } \\ \delta_{m} & \text { Gauss weights } \\ \varphi_{l} & \text { spatial abscissa }\end{array}$




\section{Subscripts}

$\partial \mathcal{D}_{e} \quad$ bounded domain of the $2 \mathrm{D}$ enclosure

$\partial \mathcal{D}_{i} \quad$ bounded domain of the rectangular obstacle

$\{\mathcal{D}\} \quad$ bounded domain of the participating medium in $\mathbb{R}^{2}$

\section{Introduction}

The conductive-radiative heat transfer in participating medium keeps getting attention of researchers, because it is encountered in various engineering applications such as heat transfer in the semi-transparent media, glass fabrication, fibrous thermal insulation, porous materials, industrial furnaces [1-3]. Hereafter, thermal radiation investigated in the present paper is applied within a cavity of complex or irregular shape at high level of temperature. Radiation contributes in the heat transfer because of absorption and emission processes inside the participating medium.

Up to now, an exact analytical solution of radiative transfer equation (RTE) is difficult to find for multi-dimensional complex geometries, but remains in strong demand to solve various thermal problems [2]. Numerous studies dealing with combined conduction-radiation heat transfer have already been examined, whose implementation was realized in the case of simple and complex geometries by using numerical techniques. Mostly, numerical methods used included various schemes such as the zonal method, the Monte Carlo method, the spherical harmonic method, the discrete ordinates method (DOM), the finite-volume method (FVM), the finite element method (FEM), the ray tracing/nodal-analyzing method (RTNAM) and the Discontinuous Galerkin method (DG).

In simple geometries, such as square enclosures containing semi-transparent medium, Yuen and Takara [1] considered a combined conductive-radiative heat transfer in a two dimensional enclosure with a gray medium. The authors applied a numerical procedure, based on tabulated values from a class of generalized exponential integral function $S_{n}(x)$, known to be particularly accurate and efficient in the case of pure radiation $[4,5]$. Numerical data of temperature and flux fields were tabulated as benchmark solutions of the coupled problem, since exact analytical solutions are unable to obtain except for restricted one-dimensional problems. The authors successfully shown that for some ranges of optical thicknesses and conduction-radiation parameters, the diffusion approximation commonly used in practical engineering problems yields to significant errors on radiative quantities. Kim and Baek [2] studied the same problem by using the DOM combined to a diamond spatial scheme for the RTE, whereas the energy equation was discretized with a central finite differences scheme. Their results were compared with the literature in good agreement. Lee and Viskanta [3] investigated a combined conductive-radiative heat transfer by comparing the solutions in the case of a two-dimensional semi-transparent medium. The authors used the DOM and the Rosseland diffusion approximation to solve the RTE. The 
authors claimed that, the results predicted by the DOM are in good agreement compared with those based on the 1D integral formulation but suffers from the numerical smearing. In the other hand, the diffusion approximation greatly underpredicts the radiative quantities; especially for optically thin medium. Mishra et al. [6] used the Lattice Boltzmann Method (LBM) to solve the conductionradiation energy equation inside a two-dimensional rectangular enclosure. The authors examined the case of a gray absorbing, emitting and scattering participating medium, enclosed inside a single cavity without inner obstacle. The FVM and LBM where applied to solve the energy equation, while the RTE was solved by using the collapse dimension method (CDM). The results of dimensionless temperature distributions were plotted, and the number of required iterations to reach steady-state solutions between the LBM and the FVM where compared. It was observed that the LBM converged faster than FVM in case of coupled radiation-diffusion. In fact, researchers such as Sakami et al. [7], Rousse et al. [8], Mahapatra et al.[9], Luo and Shen [10], Lazard [11], Ghattassi et al.[12], Keshtkar [13], have developed similar analysis with excellent results compared to benchmarks solutions, when the size of the obstacle is set to be zero.

For complex or irregular geometries shapes such as rectangular shaped geometries, Asllanaj et al. [14] analysed a transient conduction-radiation combined heat transfer for a gray absorbingemitting medium in a 2D cavity modelled with unstructured triangular meshes. The RTE has been solved with both a new FVM based on vertex scheme and a modified exponential scheme. Amiri et al. [15] studied a combined conductive-radiative heat transfer problem, applied to irregular geometries containing an anisotropic scattering participating medium. The authors used the FVM to solve the energy equation and the DOM to solve the radiative transfer equation. The results in terms of dimensionless temperature and radiative flux were compared to benchmark with a good accuracy. They also kindly discussed the influence of conduction-radiation parameters and optical properties on the values of radiative quantities. Lari and Gandjalikhan Nassab [16] analysed the combined radiative-conductive heat transfer in 3D complex geometries by using a block-off method. As applied in CFD even in two-dimensions, the FVM was used to solve the gas energy equation while the DOM was used to solve the RTE in the absorbing-emitting and linear anisotropic scattering medium. The results of the model were validated, and compared to the literature then, applied to analyze the effects of conduction-radiation parameters and radiative properties on the temperature and flux fields in the medium. Talukdar et al. [17] discussed on a new technique to perform combined conduction-radiation heat transfer in a complex porous structure. FVM and block-off methods based on Cartesian coordinates were used to evaluate the effective thermal conductivity for different temperature, emissivity, thermal conductivity of the solid and absorption coefficient of the fluid. The results of dimensionless temperature and heat flux distributions were compared to those obtained when using FLUENT 6.3, and remained in good agreement. Zare and Gandjalikhan Nassab [18] investigated the radiative-conductive heat transfer problem with a variable thermal conductivity inside 2D complex geometries. In fact, the FVM was used to solve the energy equation whereas the discrete ordinates method has been used to solve the RTE, by deducing the temperature and heat flux distributions in the medium. The blocked-off procedure in Cartesian coordinates has also been employed to treat both straight and curvilinear boundaries of the concerned geometries. The effects of a variable thermal conductivity, the non-uniformity of boundary heat fluxes due to the isotherm obstacle, both on temperature and heat flux distributions have been analysed and carefully discussed. Keshtkar and Amiri [19] simulated the combined radiative-conductive heat transfer in a $2 \mathrm{D}$ enclosure containing an isotherm rectangular obstacle located at the cavity's center. The RTE and energy equation was solved by using the DOM and LBM respectively. The authors discussed how the radiative 
properties, the conduction-radiation parameters, and the isotherm obstacle modify the temperature and heat flux distributions. Studies involving coupled radiation-convection inside rectangular enclosures with obstacles [20-21] are also available, for which similar methods as examined previously were implemented.

From the literature survey dealing with the coupled conductive and radiative heat transfer within multi-dimensional complex geometries, mostly numerical methods have been developed to solve the problem. Moreover, the aim of the present paper is to establish an exact semi-analytical solution of the conductive-radiative heat transfer problem, applied to a $2 \mathrm{D}$ absorbing and emitting medium with a centered square obstacle. Exact expressions of the incident radiation from the RTE as developed in the case of radiation heat transfer [22,23] are set and coupled with energy equation by using a centered finite scheme in order to derive the temperatures and heat flux distributions. An adequate numerical algorithm to solve the considered conductive-radiative problem is given and performed to obtain the numerical results of temperature and heat flux.

\section{Geometry}

One considers in the Fig. 1 a the semi-transparent medium enclosed in a two dimensional square cavity of length $H$, containing a square obstacle of length $h$ at a center position. From Djeumegni et al. [23], analytical expressions of the radiative quantities examined in this paper are those derived for the case $H=3 h$. Modelling involving geometries for which $H<3,3 h<H \leq$ $(2+\sqrt{5}) h$ and $H>(2+\sqrt{5}) h$ are not developed here for conciseness. However, some numerical results and simulations are presented in the cases of $H=(2+\sqrt{5}) h$ and $H=8 h$. The bounded domain of the $2 \mathrm{D}$ enclosure denoted by $\partial \mathcal{D}_{e}$ and the one for the obstacle denoted by $\partial \mathcal{D}_{i}$ are both kept at constant imposed temperature $T_{r e f}$. Thus, the bounded domain concerning the participating medium denoted by $\{\mathcal{D}\}$ is defined in the $(x, y)$ plane by:

$$
\{\mathcal{D}\}=\left\{(x, y) \in[0, H] \times[0, H] \backslash(] \frac{H-h}{2}, \frac{H+h}{2}[\times] \frac{H-h}{2}, \frac{H+h}{2}[)\right\} .
$$

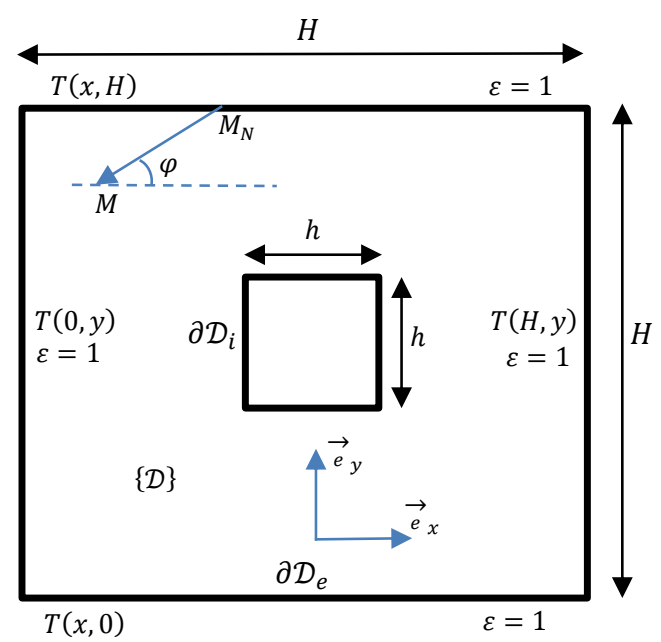

Fig.1. Section of the semi-transparent medium in $(x, y)$ reference. 
Modelling of the combined heat transfer is done in the same plane $(x, y)$, implying no variation of radiative quantities along the $z$-axis.

The incoming intensity depends of the direction vector $\vec{\Omega}=\left(\begin{array}{c}\cos \varphi \sin \theta \\ \sin \varphi \sin \theta \\ \cos \theta\end{array}\right)$, with $\varphi \in[0,2 \pi]$ and $\theta \in[0, \pi]$. So, in the plane $(x, y)$ the third $\operatorname{component} \cos \theta$ is not taken in consideration but must not be forgotten in the integrated radiative quantities.

\section{Mathematical formulation}

\subsection{Conductive heat transfer}

The energy equation for a transient combined conductive-radiative heat exchange without external heat source is written:

$$
\rho C_{p} \frac{\partial T(t, s)}{\partial t}=-\vec{\nabla} \cdot\left(\vec{q}^{r}(t, s)+\vec{q}^{c}(t, s)\right)
$$

where $\rho$ is the mass density of the medium and $C_{p}$ its specific heat. The different thermo-physical properties are assumed constant and the conductive heat flux is given from the Fourier's law as:

$$
\vec{q}^{c}(t, s)=-k_{c} \vec{\nabla} T(t, s)
$$

where, $k_{c}$ is the thermal conductivity of the participating medium.

On the other hand, the radiative source term is expressed by:

$$
S^{r}(t, s)=-\vec{\nabla} \cdot \vec{q}^{r}(t, s),
$$

whence Eq. (2) is rewritten:

$$
\rho C_{p} \frac{\partial T(t, s)}{\partial t}-k_{c} \Delta T(t, s)=S^{r}(t, s)
$$

for

$$
(t, s) \in[0, s] \times\{\mathcal{D}\}
$$

in addition $\quad T(0, s)=u_{0}(s)$, for $s \in\{\mathcal{D}\}$.

where $u_{0}$ is the temperature field at the boundary. From Eq. (4), temperature distribution $T(t, s)$ remains the major unknown. Hence, to overcome with this issue, the radiative source term $S^{r}(t, s)$ in term of temperature is needed solve Eq. (5), so it will be computed from the RTE in the next subsection. 


\subsection{Radiative Heat transfer}

In [23], it has been discussed that the present geometry $(H=3 h)$ reveals the existence of sixteen sub-domains, divided into four squares and twelve triangles as illustrated in Fig. 2-(b), where the different expressions of the useful radiative quantities to evaluate have not the same analytical expressions. Assuming a uniform grid representation of the domain, the latter one is divided into $N_{x} \times N_{y}$ cells characterized either by their corresponding space coordinates $\left(x_{i}, y_{j}\right)$ or their nodes labelled $(i, j)$ in order to discretize the needed equations. Therefore, any point $M_{i, j}$ of the participating medium is located by its discrete coordinates $\left(x_{i}, y_{j}\right)=[(i-1) \Delta x,(j-1) \Delta y]$, with a constant step length following $x$-axis given by $\Delta x=x_{i}-x_{i-1}$, while the one for $y$-axis is $\Delta y=$ $y_{j}-y_{j-1}$.

Radiation from a given point inside the semi-transparent medium that reaches any point $M \in\{\mathcal{D}\}$ follows a path length supported by the direction vector $\vec{\Omega}$. In the present paper, as illustrated in the Fig. 2-(a), only the bottom surface of the square section is hot at temperature $T_{r e f}$ and the other surfaces are set to be cold. As shown Fig. 2-(a), the radiation travelling from the bottom boundary surface up to the point $M$ follows the path of length $\delta$ such that $\overrightarrow{M_{S} M}=\delta \vec{\Omega}$. Similarly, any point on the same ray is characterized by a path length $\delta^{\prime}$ given by $\overrightarrow{M^{\prime} M}=\delta^{\prime} \vec{\Omega}$. In the reference plane $(x, y)$, one has $\delta=-y / \cos \varphi \sin \theta$ and $\delta^{\prime}=\left(y^{\prime}-y\right) / \cos \varphi \sin \theta$.

The spatial relation linking any emissive point $M^{\prime}$ within the participating medium and the calculation point $M$ on a same path for a given propagation direction, strongly depends on the angle $\varphi$ and its positions $\delta_{m} \in[0,1]$. One needs to calculate the temperature and the radiative flux at each node $(i, j)$, hence, $x_{i}^{\prime}-x_{i}= \pm \delta_{m} y_{j} \tan \varphi$ and $y_{j}^{\prime}=\left(1-\delta_{m}\right) y_{j}$ where $\left(x_{i}^{\prime}, y_{j}^{\prime}\right) \in\{\mathcal{D}\}$.

(a)

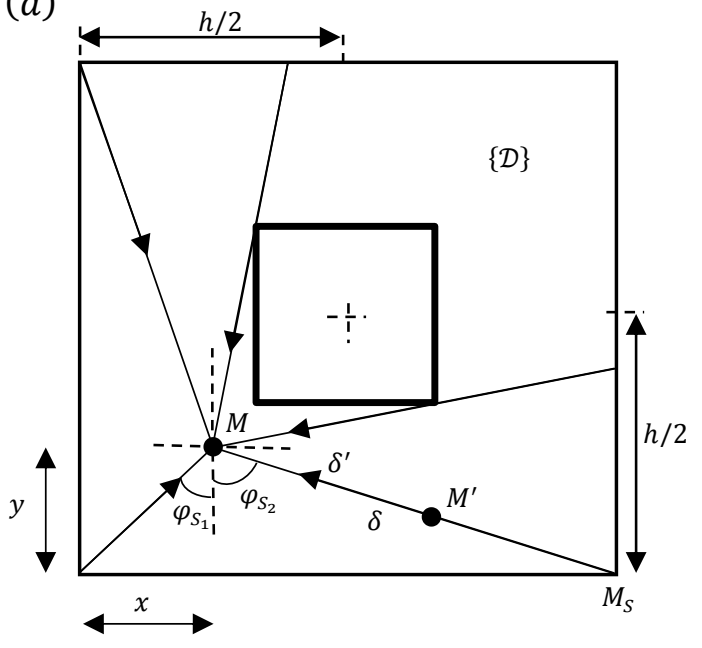

(b)

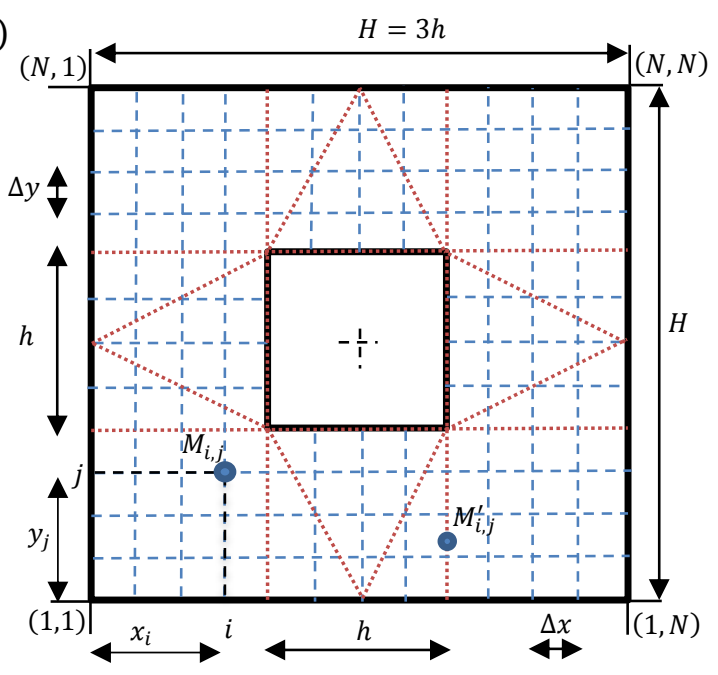

Fig. 2. (a) Ray propagation within the semi-transparent medium, (b) mesh grid within the semi-transparent medium. 
Therefore, following a similar procedure developed in [22, 23], all the locations of the calculation points in the medium are characterized by their Cartesian coordinates $\left(x_{p}, y_{q}\right)$. One has $x_{p}-\Delta x \leq$ $x_{i}^{\prime} \leq x_{p}+\Delta x$ and $y_{q}-\Delta y \leq y_{j}^{\prime} \leq y_{q}+\Delta y$, where $x_{p}=(p-2) \Delta x$, and $y_{q}=(q-2) \Delta y$. Since, $x_{i}^{\prime}$ and $y_{j}^{\prime}$ are respectively linked to $x_{i}$ and $y_{j}$, one obtains the integers $(p, q)$ in terms of $(i, j)$ as:

$$
\left\{\begin{array}{l}
p=E\left[(i+1) \pm(j-1) \delta_{m} \tan \varphi\right] \\
q=E\left[\left(1-\delta_{m}\right)(j-1)+2\right]
\end{array}\right.
$$

where, $E(a)$ denotes the integer part of the real $a$.

The RTE in its general form for a unit refractive index is written:

$$
\begin{aligned}
& \frac{1}{c} \frac{\partial I(t, s, \Omega)}{\partial t}+\vec{\Omega} \cdot \vec{\nabla} I(t, s, \Omega)=-k_{a}\left\{I(t, s, \Omega)-I_{b}[T(t, s)]\right\}, \\
& \text { with }(t, s, \Omega) \in[0, s] \times\{\mathcal{D}\} \times C^{\Omega},
\end{aligned}
$$

for boundary conditions:

$$
I(t, s, \Omega)=I_{b}[T(t, s)], \text { for }(t, s, \Omega) \in[0, \varsigma] \times\left\{\partial \mathcal{D}_{e} \cup \partial \mathcal{D}_{i}\right\} \times C^{\Omega},
$$

where, $\varsigma$ is a given time, $k_{a}$ denotes the absorption coefficient of the medium, $c=$ $2.99793010^{8} \mathrm{~ms}^{-1}$ in the vacuum and $I_{b}[T]$ is the radiation intensity of the blackbody, depending of transient temperature in the medium and expressed by:

$$
I_{b}[T]=\frac{\sigma_{B} T^{4}}{\pi},
$$

where, $\sigma_{B}=5.6698 \times 10^{-8} W \cdot m^{2} \cdot K^{-4}$ is the Stefan-Boltzmann constant. Among the main applications in the semi-transparent media, the radiative propagation time is much faster compared to the thermal response of the medium, consequently $\frac{1}{c} \frac{\partial I(t, s, \Omega)}{\partial t} \approx 0$. Then, although temperature may vary slowly with time in the medium, the radiation is considered at steady state.

The incoming radiation, for a given direction of propagation $\vec{\Omega}$ is given by:

$$
I(t, s, \Omega)=\frac{\sigma_{B} T_{r e f}^{4}}{\pi} e^{-k_{a} . \delta(s, \Omega)}+\frac{k_{a}, \sigma_{B}}{\pi} \int_{0}^{\delta} T^{4}\left(t, s^{\prime}\right) e^{-k_{a} \delta^{\prime}\left(s^{\prime}, \Omega\right)} d \delta^{\prime},
$$

where the boundary is at a prescribed temperature $T_{r e f}$. The incident radiation at any point $M(x, y) \in\{\mathcal{D}\}$ at a given instant $t$ defined as: 


$$
G(t, s)=\int_{\Omega=4 \pi} I(t, s, \Omega) d \Omega,
$$

and the corresponding radiative heat flux is written:

$$
\vec{q}^{r}(t, s)=\int_{\Omega=4 \pi} I(t, s, \Omega) \vec{\Omega} d \Omega
$$

The radiative flux field divergence is classically given by:

$$
S^{r}(t, s)=k_{a}\left(G(t, s)-4 \pi I_{b}[T(t, s)]\right) .
$$

Therefore, in order to combine radiation and conduction, incident radiation must be evaluated from radiative transfer equation.

\subsubsection{Incident radiation}

The semi-analytical formulation of incoming radiation developed actually will enable to combine it with energy equation given at Eq. (5), since they are strongly coupled by the incident radiation $G(t, s)$ and the temperature distribution $T(t, s)$.

From Eq. (10), at instant t, when only the bottom boundary surface is hot at imposed temperature $T_{\text {ref }}$, and the others are cold, the incoming radiation is expanded in $\left(\vec{e}_{x}, \vec{e}_{y}\right)$ plane by:

$$
\begin{gathered}
I(t, x, y, \theta, \varphi)=\frac{\sigma_{B} T_{r e f}^{4}}{\pi} e^{-k_{a}\left\{\frac{y}{\sin \varphi \sin \theta}\right\}}+\frac{n^{2} k_{a} \sigma_{B}}{\pi} \int_{y^{\prime}=0}^{y^{\prime}=y} T^{4}\left(t, x^{\prime}, y^{\prime}\right) e^{-k_{a}\left\{\frac{y-y^{\prime}}{\sin \varphi \sin \theta}\right\}} \frac{d y^{\prime}}{\sin \varphi \sin \theta}, \\
\bullet \quad \forall(t, x, y) \in([0, \varsigma]) \times(] 0, \frac{H-h}{2}[\times] 0, \frac{H-h}{2}[),
\end{gathered}
$$

The transient incident radiation at a time $\mathrm{t}$ is deduced using the relation:

$$
G(t, x, y)=2 \int_{\varphi_{1}}^{\varphi_{2}} \int_{\theta=0}^{\theta=\frac{\pi}{2}} I(t, x, y, \theta, \varphi) \sin \theta d \theta d \varphi
$$

with, $\varphi_{s_{1}}=\tan ^{-1}\left\{\frac{x}{y}\right\}$ and $\varphi_{s_{2}}=\tan ^{-1}\left\{\frac{H-x}{y}\right\}$.

Following a similar procedure proposed in [22, 23], a first set of Bickley-Naylor functions [24] is introduced and set by:

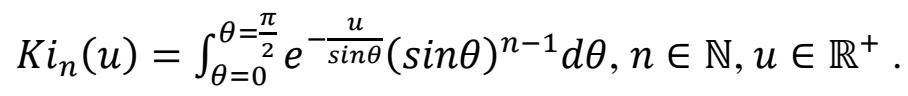

In order to eliminate the variable $\theta$ in the first integrals of Eq. (14), The Altaç functions [24] are introduced to eliminate the $\varphi$ dependence in Eq. (10) and expressed for $n \in \mathbb{N}, u \in \mathbb{R}^{+}$by:

$$
\operatorname{Bis}_{n}(u, \theta)=\int_{\varphi=0}^{\varphi=\theta} K i_{n}\left(\frac{u}{\cos \varphi}\right)(\cos \varphi)^{n-2} d \varphi
$$


For any point $M(x, y)$ located in the square sub-domain sharing the left and south boundary surfaces of the participating medium, the exact dimensionless expression of the incident radiation $G_{i, j}^{*}$ for rays covering aperture $\varphi_{s_{1}}+\varphi_{S_{2}}$ is calculated. Its discretized form when using a regular grid with square cells $\left(\Delta x^{*}=\Delta y^{*}\right)$ is then given by:

$$
\begin{aligned}
G_{i, j}^{*} & =\frac{1}{2 \pi}\left\{\operatorname{Bis}_{2}\left(k_{a}(j-1) \Delta y^{*}, \tan ^{-1}\left\{\frac{i-1}{j-1}\right\}\right)+B i s_{2}\left(k_{a}(j-1) \Delta y^{*}, \tan ^{-1}\left\{\frac{N-i+1}{j-1}\right\}\right)\right\}, \\
& +\frac{k_{a}}{2 \pi}(j-1) \Delta y^{*} \tan ^{-1}\left\{\frac{i-1}{j-1}\right\} \sum_{l=1}^{N_{\varphi}} \omega_{l} \sum_{m=1}^{M} \frac{\omega_{m}}{\cos \varphi_{l_{1}}} T_{k, p, q}^{* 4} K i_{1}\left(\frac{k_{a}(j-1) \Delta y^{*} \delta_{m}}{\cos \varphi_{l}}\right), \\
& +\frac{k_{a}}{2 \pi}(j-1) \Delta y^{*} \tan ^{-1}\left\{\frac{N-i+1}{j-1}\right\} \sum_{l=1}^{N_{\varphi}} \omega_{l} \sum_{m=1}^{M} \frac{\omega_{m}}{\cos \varphi_{l_{1}}} T_{k, p, q}^{* 4} K i_{1}\left(\frac{k_{a}(j-1) \Delta y^{*} \delta_{m}}{\cos \varphi_{l}}\right)
\end{aligned}
$$

with, $\varphi_{l_{1}}=\beta_{l} \times \tan ^{-1}\left\{\frac{i-1}{j-1}\right\}$ and $\varphi_{l_{2}}=\beta_{l} \times \tan ^{-1}\left\{\frac{N-i+1}{j-1}\right\}$ where, $\beta_{l}$ is the angular abscissa, $\omega_{l}$ and $\omega_{m}$ are the Gauss weights, $k$ is a time index, and $N_{\varphi}$ denotes the order of the Gauss quadrature used to approximate the respective integrals.

When a point $M(x, y)$ belongs to the other triangles or square subdomains delimited by broken red lines in Fig. 2-(a), incident radiation have different analytical expressions due to the geometry in the medium. For any point in the domain such that, \{] $2, N_{x}-1[\times] 2, N_{y}-1[\} \backslash\{] 2, E\left(\frac{H-h}{2 \Delta x}\right)[\times$ ] $2, E\left(\frac{H-h}{2 \Delta y}\right)[\}$, the associated equations, are not presented there for conciseness, but can be denoted as $\left(G_{l}^{*}\right)_{i, j}$, where $l \in\{1,2, \ldots 20\}$ represents the number of subdomains that contribute to the combined heat transfer.

Once $G$ is calculated for all the grid's cells including the boundary surfaces, Eqs. (12-13) are used to compute the radiative heat flux vector $\vec{q}^{r}$ and the radiative source $S^{r}=-\vec{\nabla} \cdot \vec{q}^{r}$.

\subsection{Combined conductive-radiative heat transfer}

Inserting the radiative source $S^{r}$ from (13) into Eq. (5) leads to the general energy equation which depends on the incident radiation at the calculation point:

$$
\left\{\begin{array}{c}
\rho C_{p} \frac{\partial T(t, s)}{\partial t}-k_{c} \Delta T(t, s)=k_{a}\left(G(t, s)-4 \pi I_{b}[T(t, s)]\right), \\
T(0, s)=v_{0}(s)
\end{array}\right.
$$

for

$$
(t, s) \in[0, s] \times\{\mathcal{D}\}
$$

where, $v_{0}$ denotes the temperature field at a boundary surface for $t=0$. Eq. (18) is modified and converted into dimensionless form, by using some dimensionless parameters detailed in Appendix A. Consequently, one replaces each of dimensionless parameters set above in Eq. (19), that 
characterizes the transient heat transfer by conduction and radiation within each subdomain located by the label $l$ as:

$$
\left\{\begin{array}{c}
\frac{\partial T_{l}^{*}\left(\xi, s^{*}\right)}{\partial \xi}-\Delta T_{l}^{*}\left(\xi, s^{*}\right)=\eta\left(G_{l}^{*}\left(\xi, s^{*}\right)-T_{l}^{* 4}\left(\xi, s^{*}\right)\right)=\eta S_{l}^{r^{*}}, \\
T_{l}^{*}\left(0, s^{*}\right)=v_{l, 0}^{*}\left(s^{*}\right),
\end{array}\right.
$$

where, $\eta=k_{a}^{2} H^{2} / N_{p l}$. When $\partial \xi \rightarrow \infty$, the steady state is reached and one obtains:

$$
\Delta T_{l}^{*}\left(s^{*}\right)=\eta\left(T_{l}^{* 4}\left(s^{*}\right)-G_{l}^{*}\left(s^{*}\right)\right)=-\eta S_{l}^{r^{*}}
$$

\subsubsection{Semi-analytical solution of combined conductive-radiative heat transfer}

Several numerical methods already exist to solve this problem dealing with combined radiation and conduction. In this paper, one implements a discrete finite differences scheme regarding its simplicity and accuracy on results.

One discretizes uniformly the time domain $\xi \geq 0$ by setting $\Delta \xi=\xi_{k+1}-\xi_{k}$ with $\xi_{k}=k \Delta \xi$ for any $k \in\left[0, N_{t}\right]$, where $N_{t}$ is the maximum number of time steps.

\section{- Temperature distribution}

The temperature distribution is evaluated using a finite difference method and Dirichlet boundary condition applied to the dimensionless energy equation. At time index $k$, and at node labelled $(i, j)$, Eq. (19) is rewritten as:

$$
\left(\frac{\partial T_{l}^{*}}{\partial \xi}\right)_{i, j}^{k}-\left[\left(\frac{\partial^{2} T_{l}^{*}}{\partial x^{* 2}}\right)_{i, j}^{k}+\left(\frac{\partial^{2} T_{l}^{*}}{\partial y^{* 2}}\right)_{i, j}^{k}\right]=\eta\left[\left(G_{l}^{*}\right)_{i, j}^{k}-\left(T_{l}^{* 4}\right)_{i, j}^{k}\right]
$$

The left side of Eq. (22) is approximated by a finite differences method. The temperature is then approximated by a first order time forward difference method and set as:

$$
\left(\frac{\partial T_{l}^{*}}{\partial \xi}\right)_{i, j}^{k} \approx \frac{\left(T_{l}^{*}\right)^{k+1}-\left(T_{l}^{*}\right)^{k}}{\Delta \xi}+0(\Delta \xi)
$$

where, $\Delta \xi=\xi / N_{t}$.

The second order of the partial differential equation, which represents the spatial variation of temperature in the $(x, y)$ reference is also given when using a central finite differences scheme as:

$$
\left(\frac{\partial^{2} T_{l}^{*}}{\partial x^{* 2}}\right)_{i, j}^{k} \approx \frac{\left(T_{l}^{*}\right)_{i-1, j}^{k}-2\left(T_{l}^{*}\right)_{i, j}^{k}+\left(T_{l}^{*}\right)_{i+1, j}^{k}}{\left(\Delta x^{*}\right)^{2}}+0\left[\left(\Delta x^{*}\right)^{2}\right]
$$




$$
\left(\frac{\partial^{2} T_{l}^{*}}{\partial y^{* 2}}\right)_{i, j}^{k} \approx \frac{\left(T_{l}^{*}\right)_{i, j-1}^{k}-2\left(T_{l}^{*}\right)_{i, j}^{k}+\left(T_{l}^{*}\right)_{i, j+1}^{k}}{\left(\Delta y^{*}\right)^{2}}+0\left[\left(\Delta y^{*}\right)^{2}\right]
$$

where, $\Delta x^{*}=\Delta x / H$ and $\Delta y^{*}=\Delta y / H$.

Substituting Eq. (23-25) into Eq. (22), implies:

$$
\begin{aligned}
\frac{\left(T_{l}^{*}\right)^{k+1}-\left(T_{l}^{*}\right)^{k}}{\Delta \xi} & -\left[\frac{\left(T_{l}^{*}\right)_{i-1, j}^{k}-2\left(T_{l}^{*}\right)_{i, j}^{k}+\left(T_{l}^{*}\right)_{i+1, j}^{k}}{\left(\Delta x^{*}\right)^{2}}+\frac{\left(T_{l}^{*}\right)_{i, j-1}^{k}-2\left(T_{l}^{*}\right)_{i, j}^{k}+\left(T_{l}^{*}\right)_{i, j+1}^{k}}{\left(\Delta y^{*}\right)^{2}}\right] \\
& =\eta\left[\left(G_{l}^{*}\right)_{i, j}^{k}-\left(T_{l}^{* 4}\right)_{i, j}^{k}\right]
\end{aligned}
$$

with a truncation error of $0\left[\left(\Delta x^{*}\right)^{2} \times\left(\Delta y^{*}\right)^{2}+\Delta \xi\right]$, as shown in [26].

Once $G_{i, j}^{*}$ is evaluated, it is integrated inside the energy equation Eq. (26) in order to deduce the dimensionless temperature $\left(T^{*}\right)_{i, j}$ at the time index $k+1$. Using uniform cells, with $\Delta x^{*}=\Delta y^{*}=$ $\Delta^{*}$ and introducing the dimensionless Fourier number $F_{0}^{*}=\Delta \xi / \Delta^{* 2}$ leads to:

$$
\begin{aligned}
\left(T_{l}^{*}\right)_{i, j}^{k+1} & =F_{0}^{*}\left[\left(T_{l}^{*}\right)_{i-1, j}^{k}+\left(T_{l}^{*}\right)_{i+1, j}^{k}+\left(T_{l}^{*}\right)_{i, j-1}^{k}+\left(T_{l}^{*}\right)_{i, j+1}^{k}-\left(4-\frac{1}{F_{0}^{*}}\right)\left(T_{l}^{*}\right)_{i, j}^{k}\right] \\
& +\eta \Delta^{* 2}\left[\left(G_{l}^{*}\right)_{i, j}^{k}-\left(T_{l}^{* 4}\right)_{i, j}^{k}\right]
\end{aligned}
$$

The stability condition of this previous equation is ensured by $F_{0}^{*} \leq 1 / 4$, while the maximum number of uniform cells to used is set by $N_{x}=N_{y}=N_{\max } \leq E\left(\frac{1}{\sqrt{4 \Delta \xi}}+1\right)$, where $E$ denotes integer part of the real concerned. When the dimensionless time $\xi \rightarrow \infty$, then $F_{0}^{*} \rightarrow 1 / 4$; consequently the steady state is achieved and similar calculations yield to the same solution:

$$
\begin{aligned}
\left(T_{l}^{*}\right)_{i, j}^{k+1} & =\frac{1}{4}\left\{\left(T_{l}^{*}\right)_{i-1, j}^{k}+\left(T_{l}^{*}\right)_{i+1, j}^{k}+\left(T_{l}^{*}\right)_{i, j-1}^{k}+\left(T_{l}^{*}\right)_{i, j+1}^{k}\right\} \\
& +\eta \Delta^{* 2}\left[\left(G_{l}^{*}\right)_{i, j}^{k}-\left(T_{l}^{* 4}\right)_{i, j}^{k+1}\right]
\end{aligned}
$$

Practically, the non-linearity of Eq. (28) is analyzed using a Taylor series truncated at the linear term following the relation:

$$
\left(T_{l}^{* 4}\right)_{i, j}^{k+1}=\left(T_{l}^{* 4}\right)_{i, j}^{k}+4\left(T_{l}^{* 3}\right)_{i, j}^{k}\left[\left(T_{l}^{*}\right)_{i, j}^{k+1}-\left(T_{l}^{*}\right)_{i, j}^{k}\right]
$$

However, for one labelled subdomain of the participating medium, the dimensionless temperature appearing in Eq. (27) as the unknown variable, will be evaluated in the next section by numerical computation. A Crank-Nicolson scheme can also be involved for time discretization [12] and provides accurate solutions. Once the dimensionless temperature distribution $T_{l}^{*}$ is deduced in a 
subdomain labelled by $l$, it is used to calculate the conductive and the total heat flux distributions in the medium: thus, an iterative scheme is designed and proposed at Section 3. Consequently, one can deduce the dimensionless temperature distribution in the participating medium from the contribution of all the labelled sub-domains such that:

$$
T^{*}\left(\xi, s^{*}\right)=\sum_{1}^{20} T_{l}^{*}\left(\xi, s^{*}\right)
$$

\section{- Heat flux distribution}

The total heat flux in the medium $\vec{q}(t, s)=q_{x} \vec{\imath}+q_{y} \vec{\jmath}$ is deduced from the summation of both conductive heat flux $\vec{q}^{c}(t, s)$ and radiative heat flux $\vec{q}^{r}(t, s)$. Similarly with the expressions of radiative heat flux developed in Ref. [24] when $H=3 h$, one has the relation below:

$$
\vec{q}(t, s)=-k_{c} \vec{\nabla} T(t, s)+\sum_{l=1}^{20} \vec{q}_{l}^{r},
$$

where the radiative heat flux is calculated at internal points with the temperature equation Eq. (28) using the relation:

$$
\vec{q}_{l}^{r}=\int_{\Omega=4 \pi} I_{l}(\Omega)|\vec{\Omega} \cdot \vec{n}| d \Omega, \quad l=1, \ldots 20
$$

where $\vec{n}$ denotes the outward normal to the considered boundary surface.

In a dimensionless form, the total heat flux is set along $x$ and $y$ directions as:

$$
q_{x}^{*}=-N_{p l} \frac{\partial T^{*}}{\partial \tau_{x}}+\frac{q_{x}^{r}}{4 \sigma_{B} T_{r e f}^{4}}
$$

and,

$$
q_{y}^{*}=-N_{p l} \frac{\partial T^{*}}{\partial \tau_{y}}+\frac{q_{y}^{r}}{4 \sigma_{B} T_{r e f}^{4}}
$$

where $q_{x}^{r}$ and $q_{y}^{r}$ represent the expressions of the radiative heat flux along $x$ and $y$ axis; $\tau_{x}$ and $\tau_{y}$ denote the optical thickness of the medium along the two $x$ and $y$ directions.

\section{Numerical results}

Suitable calculations dealing with the combined conductive-radiative heat transfer in a $2 \mathrm{D}$ semitransparent medium, with a centered obstacle of length $h$ have been carried out in the present paper. Now, one intends to evaluate the accuracy of the present method by displaying numerical results both at steady and transient states. One applied it to a simple $(h=0)$ and complex $(h \neq 0)$ geometries. All calculations have been performed with a computer DELL, Intel(R) Core(TM) i78700 CPU@ 3203.19 GHz, using a FORTRAN software. 
The numerical computation follows an iterative scheme and the main steps are the following ones:

a. Initialize the temperature $T_{0}$ in the whole medium and at the boundary surfaces.

b. Solve RTE by using the exact semi-analytical method for each subdomain labelled by $l$ at each point located by $(i, j)$ following the algorithm:

- Compute the incident radiation $G_{i, j}^{*}$ from Eq. (18) at radiative equilibrium

- Evaluate the radiative heat flux $\vec{q}^{r}$ and the radiative source $S^{r}$.

c. Solve the energy equation Eq. (20) to obtain dimensionless temperature distribution $T^{*}$ from Eq. (28).

d. Repeat steps c until convergence with the criterion $\operatorname{Max}\left[\frac{\left(T_{i, j}^{*}\right)^{k}-\left(T_{i, j}^{*}\right)^{k-1}}{\left(T_{i, j}^{*}\right)^{k-1}}\right] \leq 10^{-5}$.

The number of required iterations depends on the conduction radiation parameter, the quadrature and the number of cells choice. When running the studied cases, the results of $G_{i, j}^{*}$ have been obtained with less than 500 iterations with the same criterion indicated at step d. Contrariwise for the results of $T_{i, j}^{*}$, less than 100 iterations have been useful at transient state to reach the steady one.

\subsection{Simple geometry $: h=0$}

The concerned geometry consists in a square cross section of $H \times H=1 \times 1 \mathrm{~m}^{2}$ containing an absorbing, emitting but non-scattering medium. The thermal conductivity $k_{c}$ is constant, all the boundary surfaces are black $(\varepsilon=1)$. The south boundary surface is hot, maintained at a reference constant temperature $T_{r e f}=T_{S}=100 \mathrm{~K}$ and the other one are cold at $T_{N}=T_{W}=T_{E}=T_{\text {ref }} / 2$; temperature in the medium is initially imposed to be cold at $T_{0}=T_{r e f} / 2$. With these conditions, dimensionless temperature distributions, $T^{*}=T / T_{\text {ref }}$ and heat fluxes $q^{*}=q / 4 \sigma_{B} T_{\text {ref }}^{4}$ are computed by an iterative process until convergence, then displayed and discussed.

\subsubsection{Steady-state}

The problem has already been investigated by several authors at steady-state such as Yen and Takara [1], Kim and Baek [2], Sakami et al. [7], Ismail and Salinas [25], where each of them used different methods such as FVM, finite element method (FEM), and DOM. When using the present method in the 2D enclosure, Fig. 3-(a) shows the evolution of the dimensionless temperature $T^{*}$ along the centerline position $x / H=0.5$. The results have been obtained with the conductionradiation parameters $N_{p l} \in\{0.01,0.1,1\}, H=1 \mathrm{~m}$, a unit absorption coefficient $k_{a}=1 \mathrm{~m}^{-1}$ corresponding to a unit optical thickness $\left(\tau=k_{a} H\right)$ have also been validated with the numerical solutions proposed by Mishra et al. [6] at steady state conditions. The subsequent computation has been done with a choice of $21 \times 21$ rectangular grids to facilitate the validation of results. The same Fig. 3-(a) shows a great matching between the dimensionless temperature distribution given by Mishra et al. [6], taken as benchmark results and the present one. One can argue on a good 
agreement with the literature according to the maximum relative error between the two shapes estimated at $\frac{\left|T_{\text {Mishra }}^{*}-T_{\text {Present }}^{*}\right|}{T_{\text {Mishra }}^{*}} \leq 10^{-5}$.
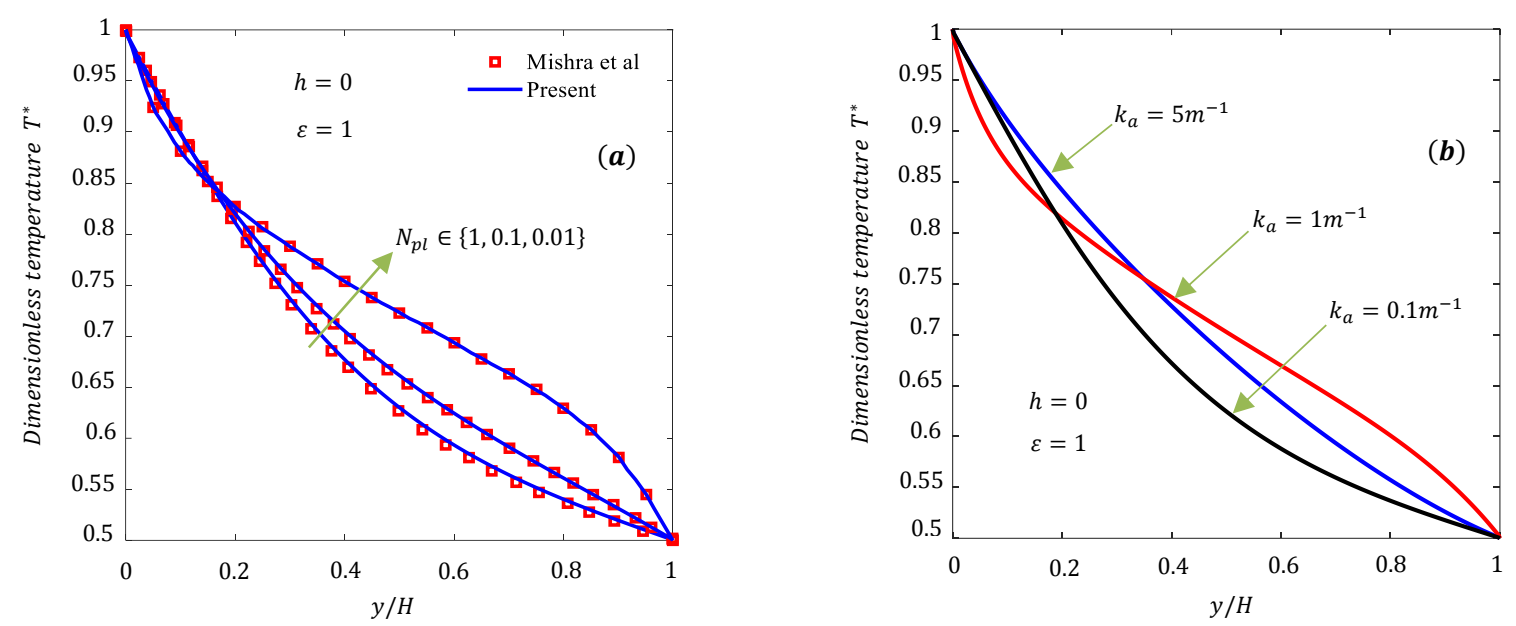

Fig.3. Dimensionless temperature at $=H / 2, h=0$ : (a) $N_{p l} \in\{1,0.1,0.01\}$ and $k_{a}=1 m^{-1}$, (b) $k_{a} \in$ $\{5,1,0.1\} m^{-1}$ and $N_{p l}=0.1$.

The respective values assigned to the conduction-radiation parameter $N_{p l}=k_{c} k_{a} /\left(4 \sigma T_{r e f}^{4}\right)$ exist to show the influence of both conduction and radiation on the temperature distribution in the participating medium. When $N_{p l}=1$, conduction predominates, hence only the hot boundary surface contribute substantially to heat transfer. The solution obtained remains similar with the case of pure conduction seen as the main mode of heat transfer. When $N_{p l}=0.1$, neither conduction nor radiation predominates one over the other. Consequently, when the Planck number $N_{p l}$ decreases and reaches 0.01 , the values of temperature increase but stay less than the case of pure radiation. In this condition, radiation heat transfer predominates over conduction. Sakami et al. [27], Rousse et al. [28], and Talukdar et al. [29] have already proven this analysis. At steady state, one can also observe on Fig. 3-(a) that, as $k_{a}$ increases, the participating medium does not enable radiations coming from boundary surfaces to penetrate in the medium. Fig. 3-(b) illustrates the effect of absorption coefficient $k_{a}$ on the dimensionless temperature profile at $x=H / 2$. One can note that when $k_{a}$ increases and reaches a large value such as $k_{a}=5 \mathrm{~m}^{-1}$ for the present case, the shape of $T^{*}$ displayed becomes similar to a straight line, whereas in the opposite direction one can observe a curvilinear shape. For this purpose, it means that the medium becomes thick and the energy generated by radiation is very quickly dissipated by absorption process. When $k_{a}=$ $0.1 \mathrm{~m}^{-1}$, the medium still participates weakly to radiative heat transfer, whereas from up to $k_{a}=$ $1 \mathrm{~m}^{-1}$ there is a strong participation.

\subsubsection{Tabulated data of conductive-radiative quantities}


In this section, the numerical results of $T^{*}$ are compared with the literature as well as the effect of cell numbers on $T^{*}$. The CPU time obtained for different values of $N_{p l}$ and $M_{\theta} \times M_{\varphi}$ is also presented.

\section{Table 1}

Comparison of dimensionless temperature $T^{*}$ at steady state for $x=H / 2$ at location $y / H \in$ $\{0.3,0.5,0.7\}$, for $\tau=1.0$.

\begin{tabular}{|c|c|c|c|c|c|c|c|}
\hline$N_{p l}$ & $y / H$ & $\mathrm{Wu}$ and $\mathrm{Ou}[30]$ & Yuen and Takara [1] & Mishra et al [31] & \multicolumn{2}{|c|}{ Mishra and Roy [32] } & Present \\
\hline & & & & & FDM-FVM & LBM-FVM & \\
\hline \multirow{3}{*}{1.0} & 0.3 & 0.733 & 0.737 & 0.737 & 0.737 & 0.737 & 0.733 \\
\hline & 0.5 & 0.630 & 0.630 & 0.630 & 0.630 & 0.630 & 0.630 \\
\hline & 0.7 & 0.560 & 0.560 & 0.564 & 0.564 & 0.564 & 0.563 \\
\hline \multirow{3}{*}{0.1} & 0.3 & 0.760 & 0.763 & 0.759 & 0.759 & 0.759 & 0.755 \\
\hline & 0.5 & 0.663 & 0.661 & 0.663 & 0.664 & 0.663 & 0.661 \\
\hline & 0.7 & 0.590 & 0.589 & 0.594 & 0.596 & 0.596 & 0.591 \\
\hline \multirow{3}{*}{0.01} & 0.3 & 0.791 & 0.807 & 0.789 & 0.782 & 0.783 & 0.789 \\
\hline & 0.5 & 0.725 & 0.726 & 0.725 & 0.726 & 0.725 & 0.726 \\
\hline & 0.7 & 0.663 & 0.653 & 0.666 & 0.676 & 0.677 & 0.662 \\
\hline
\end{tabular}

\section{Table 2}

Effects of cell numbers on dimensionless temperature $T^{*}$ at $x=H / 2, N_{p l}=0.1$, and $k_{a}=1 m^{-1}, h=0$ using quadrature $M_{\theta} \times M_{\varphi}=6 \times 6$.

\begin{tabular}{cccc}
\hline$N_{x} \times N_{y}$ & $y / H=0.2$ & $y / H=0.5$ & $y / H=0.7$ \\
\hline $81 \times 81$ & 0.81224 & 0.65977 & 0.58941 \\
$101 \times 101$ & 0.81387 & 0.65965 & 0.58935 \\
$125 \times 125$ & 0.81400 & 0.65956 & 0.58879 \\
$251 \times 251$ & 0.81542 & 0.66171 & 0.59134 \\
\hline
\end{tabular}

Table 3

Computational time to obtain a converged solution of $T^{*}$ at $h=0, k_{a}=1 \mathrm{~m}^{-1}, N_{x} \times N_{y}=251 \times 251$.

\begin{tabular}{ccccc}
\hline \multicolumn{5}{c}{ CPU time } \\
\hline & $M_{\theta} \times M_{\varphi}$ & \multicolumn{1}{c}{$N_{p l}=1$} & $N_{p l}=0.1$ & $N_{p l}=0.01$ \\
\cline { 2 - 5 }$h=0$ & $4 \times 4$ & $6 \min 45 \mathrm{~s}$ & $6 \min 45 \mathrm{~s}$ & $6 \mathrm{~min} 43 \mathrm{~s}$ \\
& $5 \times 5$ & $10 \min 2 \mathrm{~s}$ & $10 \min 8 \mathrm{~s}$ & $10 \mathrm{~min} 8 \mathrm{~s}$ \\
& $6 \times 6$ & $13 \min 53 \mathrm{~s}$ & $14 \min 6 \mathrm{~s}$ & $14 \mathrm{~min} 8 \mathrm{~s}$ \\
\hline
\end{tabular}

Table 1 highlights the values of $T^{*}$ that correspond to the absorbing-emitting medium computed when the optical thickness takes a value $\tau=1$. The results are presented at three locations along the centerline $x=H / 2$ for three values of the conduction-radiation parameter $N_{p l}$ and compared with those reported in the literature [1] and [30-32]. For all aspect ratios in the LBM+DTM, LBM+DOM, LBM+FVM with $6 \times 12$ rays used for numerical integrations, there is not a significant difference on results obtained using the present method compared with the literature. 
In this case where we are dealing with a simple geometry (without obstacle), the advantage to be noted is related to the ease of handling and computing the semi-analytical solutions of the problem.

Using the present method, Table 2 presents the effects of cell numbers on dimensionless temperature $T^{*}$ set at three locations, $y / H=0.2,0.5$ and 0.7 along the centerline $x=H / 2$. From the same table, $M_{\theta} \times M_{\varphi}=6 \times 6$ directions have been used, and there is no significant variation on results obtained for small and large values of cell numbers.

Table 3 shows the computational time taken to obtain the converged results of $T^{*}$ at steady state using the present method. The number of cells used in computation is $N_{x} \times N_{y}=251 \times 251$. For $N_{p l}=1$, there is dominance of conduction compared to radiation and since conduction is a local phenomenon, the results converge faster with almost all the values of $M_{\theta} \times M_{\varphi}$ involved in the table. In contrast to $N_{p l}=0.01$, there is dominance of radiation compared to conduction, and convergence on results is reached with a difference of $15 \mathrm{~s}$ with $M_{\theta} \times M_{\varphi}=6 \times 6$; $6 \mathrm{~s}$ with $M_{\theta} \times$ $M_{\varphi}=5 \times 5$ and $2 \mathrm{~s}$ with $M_{\theta} \times M_{\varphi}=4 \times 4$ respectively. The more the number of directions $M_{\theta} \times$ $M_{\varphi}$ increases, the more the CPU time also increases. From these tabulated results on can assert with good confidence, the accuracy and fastness of the method expanded.

\subsubsection{Numerical simulation of conductive-radiative quantities}

In this section, the numerical simulations of dimensionless temperature and heat flux are presented for different values of the conduction-radiation parameter.

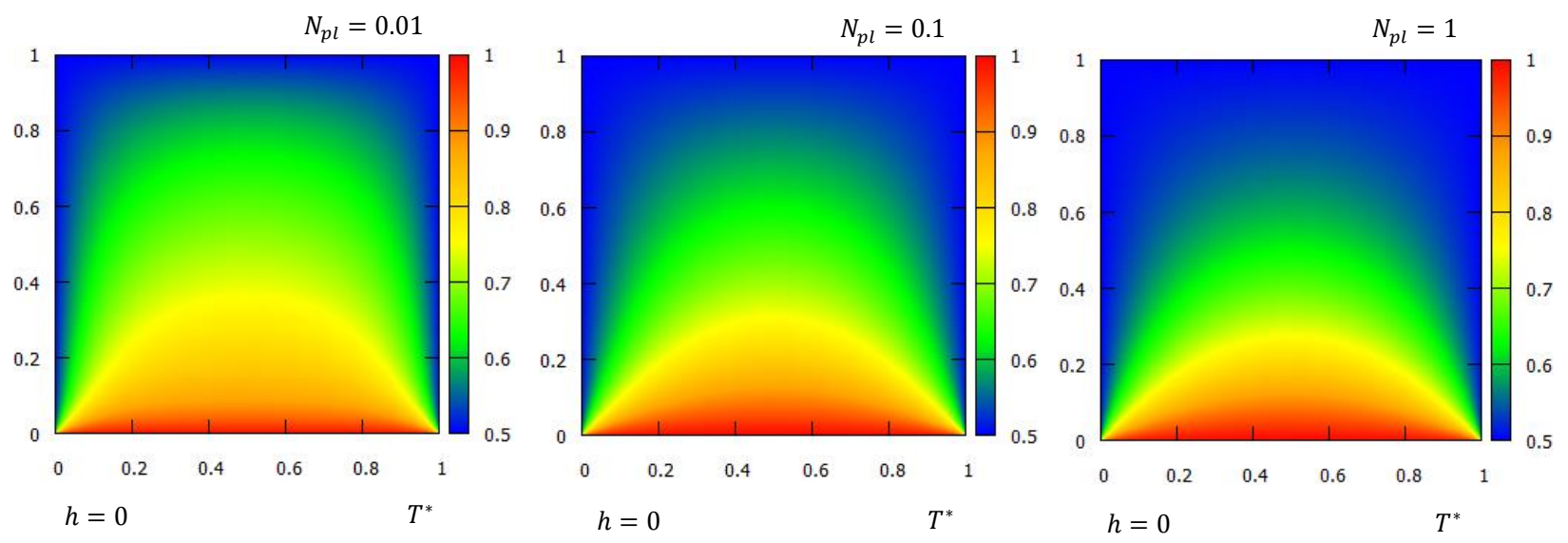

Fig.4. Dimensionless temperature distribution $T^{*}: \tau=1, \varepsilon=1, h=0, N_{p l} \in\{0.01,0.1,1\}$.

Fig.4 highlights three main numerical simulations of dimensionless temperature $T^{*}$ in a semitransparent medium enclosed in a 2D enclosure when there is no obstacle $(h=0)$ and for the values of $N_{p l}=0.01,0.1$ and 1 . The aim is to show the behavior of $T^{*}$ with the conductionradiation parameter $N_{p l}$. The respective simulations have been displayed at steady state; it enables to observe the variation of $T^{*}$ during a long time until convergence. $T^{*}$ decreases from hot to cold points belonging the semi-transparent medium. Thus, when the value of $N_{p l}$ increases, the set of 
cold points neighboring the north, est and west boundary surfaces increase, because of lack in radiation propagation. The medium absorbs more radiations and temperature distribution depends straight with the value imposed at the south boundary surface.
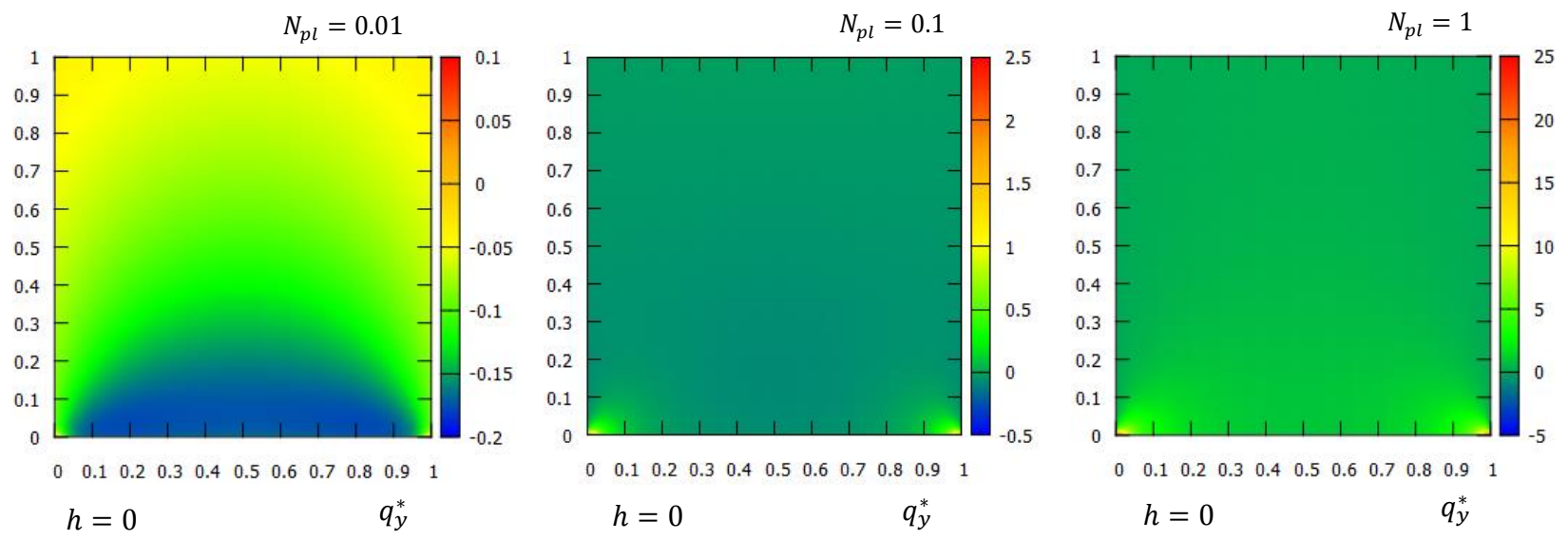

Fig.5. Dimensionless conductive-radiative heat flux $q_{y}^{*}$ following y-direction for

$$
N_{p l} \in\{0.01,0.1,1\}, \varepsilon=1, k_{a}=1 m^{-1}, h=0 .
$$

Fig. 5 shows simulations of the dimensionless conductive-radiative heat flux following $y$-direction computed for $N_{x}=N_{y}=251, \varepsilon=1, k_{a}=1 m^{-1}$, and $N_{p l} \in\{0.01,0.1,1\}$. When, $N_{p l}=0.01$ conduction in the medium remains ineffective compared to radiation. Hence, the simulation takes quietly a view similar to Fig. 8 in the Ref. [23] when $\tau=1$. At once $N_{p l}$ starts to increase from 0.01 to 1 , energy transfer becomes straightly dominated by conduction heat transfer due to the hot boundary surface. Regardless the difference on magnitude of each simulation presented, there is a symmetry with respect to the centerline position $x / H=0.5$. The numerical data of conductiveradiative heat flux obtained to display Fig. 5 using Eq. (34), can also be used to deduce the evolution of heat flux at the boundaries as already discussed in $[12,15,33]$. The authors have shown in 1-D, the heat flux by radiation and/or conduction at the hot wall of the 2-D enclosure.

\subsubsection{Transient state}

In this section, the results of $T^{*}$ obtained with the present method are compared with the literature and the numerical simulations of the dimensional temperature distribution at different times $\xi$ are presented.

\subsubsection{Numerical verification}



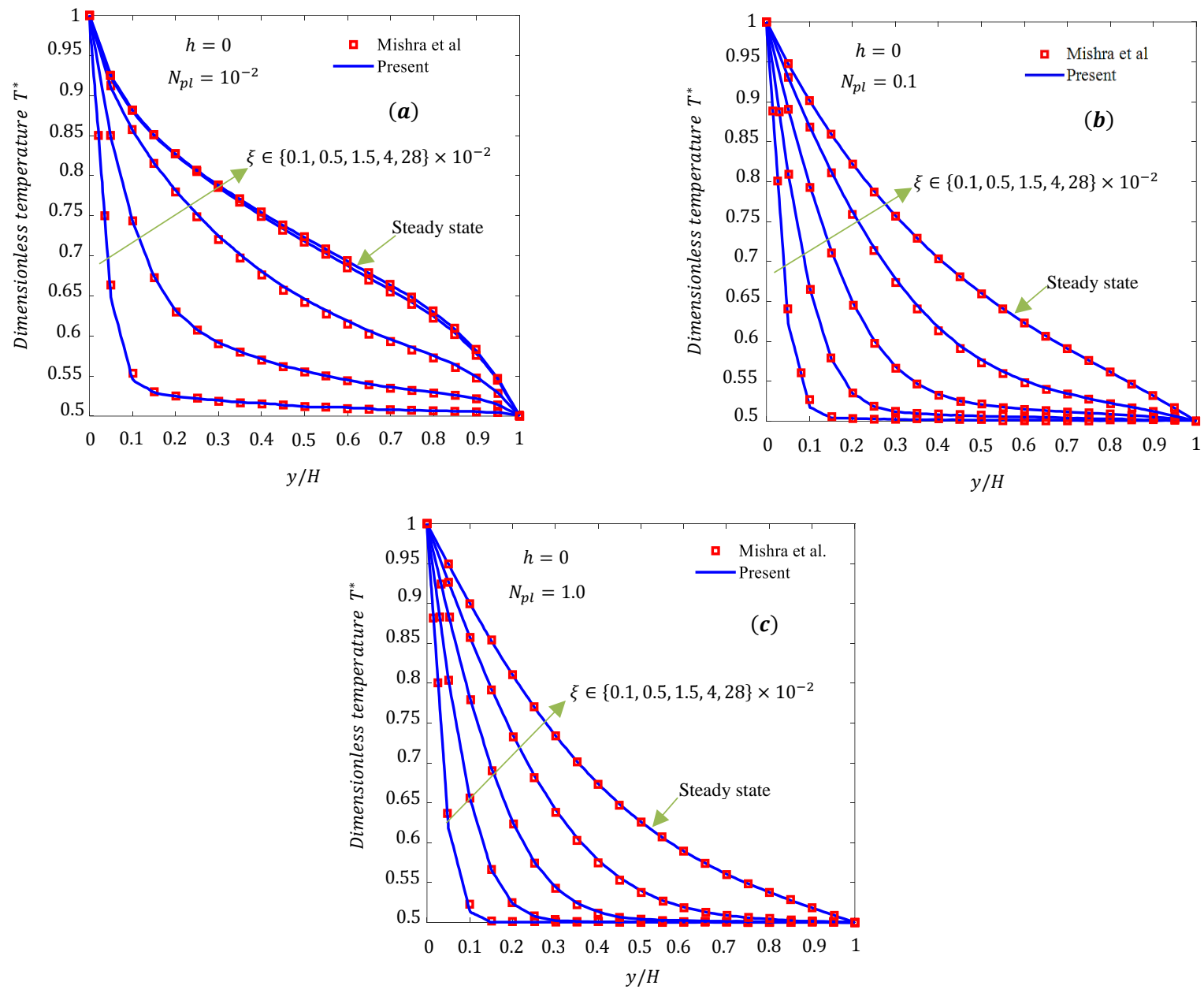

Fig.6. Dimensionless temperature $T^{*}$ in the participating medium when $h=0, k_{a}=1 \mathrm{~m}^{-1}$ at different instant, with $N_{p l} \in\{0.01,1,0.1\}$ for (a), (b) and (c) respectively.

Mishra and Lankadasu [6], have used the LBM and the collapse-dimension method (CDM) to solve energy equation and radiative information. The authors have analyzed it in a $2 \mathrm{D}$ geometry with unstructured grids, no longer with the one proposed by Mishra et al. [32] using LBM-FVM and/or FVM-FVM. It is shown on Fig. 6-(a) to Fig. 6-(c), an evolution of dimensionless temperature along the centerline $x=H / 2$ at various instants $\xi$ and for different conductionradiation parameters $N_{p l}=0.01,0.1$ and 1 . A dimensionless time step has a constant value of $\Delta \xi=5.10^{-4}$, where $\Delta \xi=\xi / N_{t}$. Steady state is reached at $\xi=28 \times 10^{-2}$ by increasing gradually the number of time index $k$; thereby one can also refer to Eq. (28) to get the same result. On the other hand, the steady-state condition has been achieved when the maximum variation of dimensionless temperature at any location between two consecutive time levels obeys to $\frac{\left|T_{\text {Mishra }}^{*} T_{\text {Present }}^{*}\right|}{T_{\text {Mishra }}^{*}} \leq 10^{-5}$. Obviously, the present results displayed on Fig. 6-(a) to Fig. 6-(c) converge suitably with those existing in the literature. According to the present study, some investigations can also be done using Dirichlet and Robin boundary conditions [12]. Indeed, the 
use of the thermal Robin boundary conditions requires the knowledge of the convective exchange coefficient $h_{c}$ of the wall; the surrounding temperature $T_{\infty}$ and the Biot number $B_{i}=\frac{h_{c} H}{k_{c}}$ and one can neglect the gain and losses by radiation for sake of complexity on equations to expand.

\subsubsection{Numerical simulations}

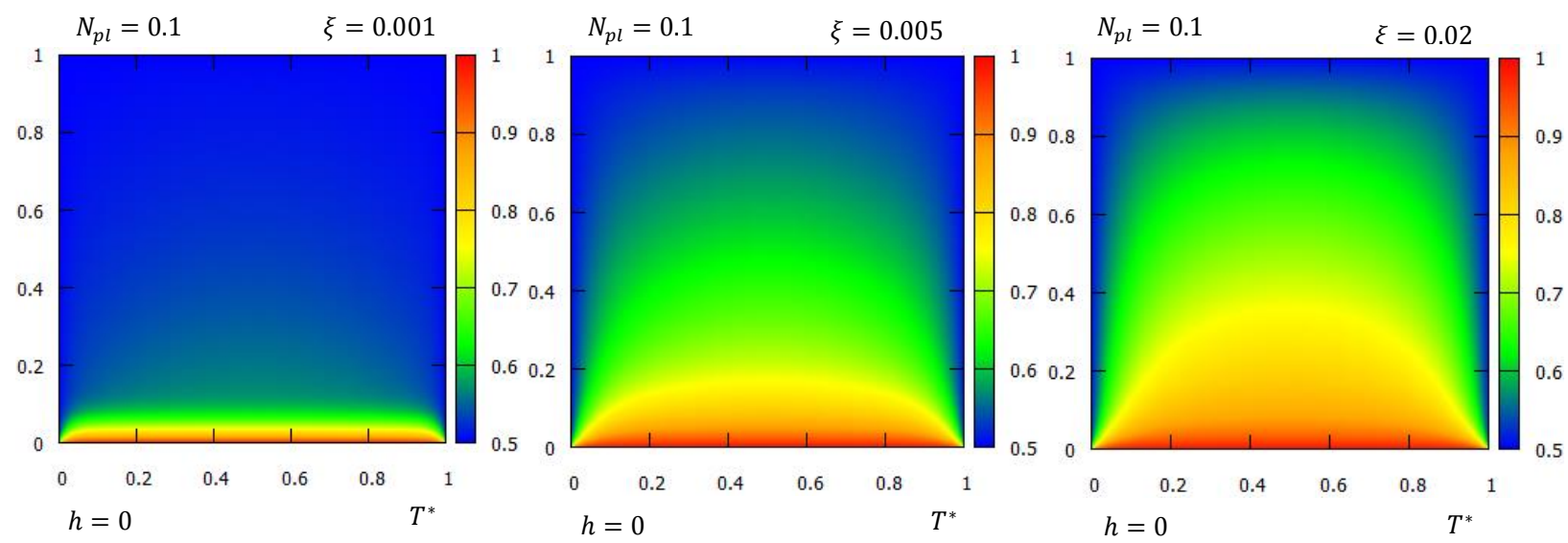

Fig.7. Transient dimensionless temperature distribution $T^{*}: \tau=1, \varepsilon=1, h=0, N_{p l}=0.1, \xi \in\{1,5,15\} \times 10^{-3}$.

In this section, three simulations of the transient dimensionless temperature distribution are presented for a simple geometry $(H=1 \mathrm{~m})$. In this case, one used a dimensionless time step $\Delta \xi=$ $10^{-6}$ and $N_{x} \times N_{y}=251 \times 251$ cells. This choice of parameters has been done in order to respect the condition of stability $F_{0} \leq 1 / 4$ in Eq. (27) and to display suitable simulations. The choice of $N_{p l}=0.1$ is only because neither radiation nor conduction predominates in the process of heat transfer. However, the simulations show that heat spreads through the medium as time increases. For $\xi=0.001$, the program takes $58 \mathrm{~s}$ to generate the results, whereas for $\xi=0.005$, it takes 4 $\min 17 \mathrm{~s}$ and for $\xi=0.015$ it takes $14 \mathrm{~min} 5 \mathrm{~s}$. Therefore, even if one increases or decreases the value of $N_{p l}$, the proposed simulations would have kept their symmetrical shapes, but with different CPU time to obtain the results.

Finally, it should be noted that the computational time needed to reach convergence using the present method as shown in Table 3 , increases when the conduction-radiation parameter $N_{p l}$ decreases. So, in contrast to Ghattassi et al. [12] where the Galerkin method has been used, less important the contribution of the radiative part, a more quickly steady state was reached.

\subsection{Complex geometry: $h \neq 0$}

In this section, one intends to present the numerical results of dimensionless temperature and heat flux involved for the conductive-radiative heat transfer. Computation has been performed at steady and transient state when $H=1 \mathrm{~m}$, to show how heat transfer propagates in the semi-transparent 
medium. The predicted dimensionless temperature $T^{*}$ and heat flux distributions have been displayed and discussed.

\subsubsection{Steady state}

In this sub-section, the impact of the obstacle, the cell numbers, the discrete directions and the conduction-radiation parameter are presented and discussed for $H=3 h$. Some numerical simulations of dimensionless temperature $T^{*}$ distribution are also given for $H=3 h, H=$ $(2+\sqrt{5}) h$ and $H=8 h$.
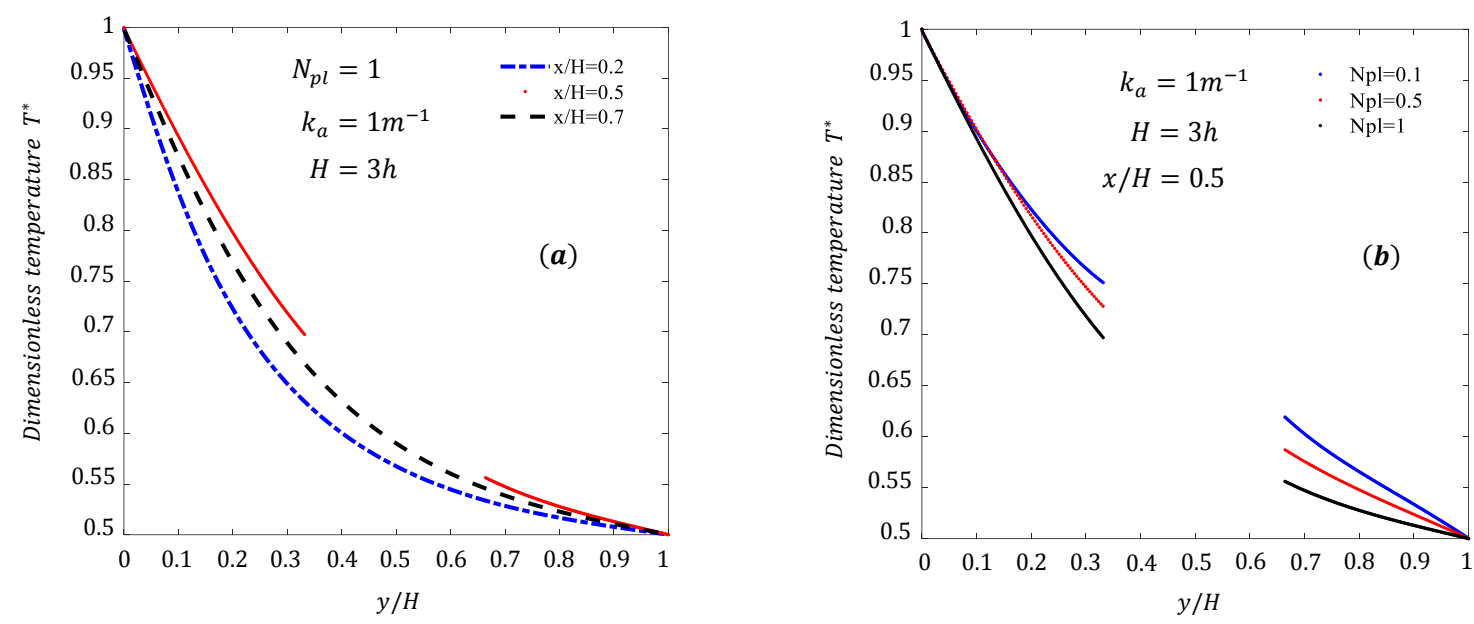

Fig.8. Dimensionless temperature profile when $H=3 h, k_{a}=0.1 m^{-1}$ : for (a) $N_{p l}=1$, $x / H \in\{0.2,0.5,0.7\}$, for (b) $N_{p l} \in\{0.1,0.5,1\}$.

On Fig. 8 , the profile of $T^{*}$ is plotted using an $M_{6} \times M_{6}$ angular discretization, additionally the boundary and initial conditions are those stated in sub-section 3.1. Hence, Fig. 8-(a) informs kindly that, the maximum values of $T^{*}$ are reached along the symmetry position $x / H=0.5$. At the same line, when $y / H \in]_{3}^{1}, \frac{2}{3}[$, the medium doesn't participate to heat transfer that is why the profile is segmented into two parts because of the centered obstacle. On the other hand Fig. 8-(b) suitably shows the effect of $N_{p l}$ on the dimensionless temperature profile at $x / H=0.5$. Higher the parameter $N_{p l}$ is and higher the decrease of $T^{*}$ and the computation time is optimized. At the center line position, when $y / H \geq 1 / 3$, the obstacle blocks any particle carrying heat from the participation and releases at $y / H \geq 2 / 3$, that is why there is an empty space when $y / H \in]_{3}^{1}, \frac{2}{3}[$. 


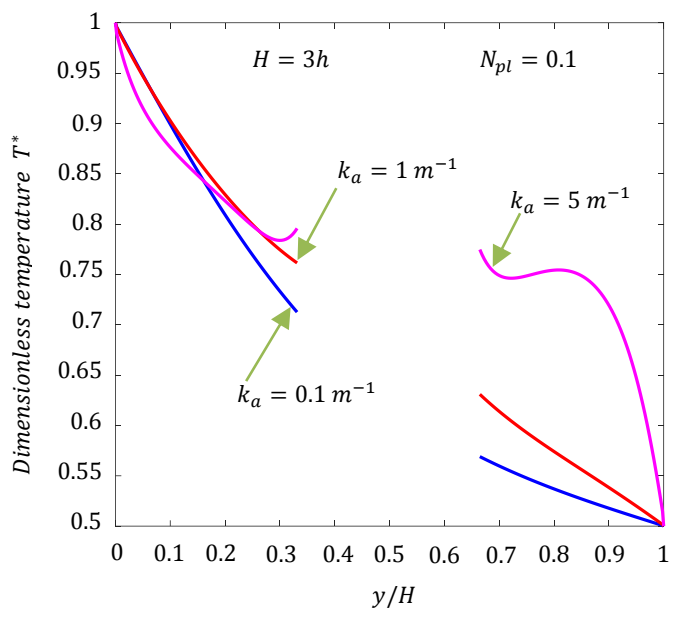

Fig.9. Dimensionless temperature $T^{*}$ along the centerline position, $x=H / 2$ for $H=3 h, k_{a} \in\{0.1,1,5\} m^{-1}$ and

$$
N_{p l}=0.1, N_{x} \times N_{y}=251 \times 251, \varepsilon=1 \text {. }
$$

Fig. 9 indicates how the obstacle modifies the temperature profile along the centerline position of the medium. For optically thick medium $\left(k_{a}=5 \mathrm{~m}^{-1}\right)$, the heat blockage effect is significant, the medium behaves like a solid semi-transparent medium, and the CPU time elapsed to reach the convergence has been $18 \mathrm{~min} 51 \mathrm{~s}$. On the other hand, when the medium becomes relatively thin (free path), the obstacle does not have a considerable influence on the heat propagation, the CPU time has decreased to $15 \mathrm{~min} 14 \mathrm{~s}$ and $13 \mathrm{~min} 7 \mathrm{~s}$ when $k_{a}=1 \mathrm{~m}^{-1}$ and $k_{a}=0.1 \mathrm{~m}^{-1}$ respectively.

\subsubsection{Tabulated data of conductive-radiative quantities}

\section{Table 4}

Effect of cell numbers on dimensionless temperature $T^{*}$ at $x / H=0.5, H=3 h, k_{a}=0.1 m^{-1}$,

\begin{tabular}{cccc}
\multicolumn{4}{c}{$N_{p l}=0.1}$. \\
\hline \multicolumn{4}{c}{$T^{*}$} \\
\hline$N_{x} \times N_{y}$ & $y / H=0.2$ & $y / H=0.7$ & $y / H=0.9$ \\
$51 \times 51$ & 0.8247 & 0.6063 & 0.5369 \\
$151 \times 151$ & 0.8260 & 0.6120 & 0.5372 \\
$251 \times 251$ & 0.8260 & 0.6110 & 0.5364 \\
\hline
\end{tabular}

\section{Table 5}

Effect of discrete directions on dimensionless temperature $T^{*}$ at $x / H=0.5, H=3 h k_{a}=0.1 m^{-1}$,

$$
N_{p l}=0.1, N_{x} \times N_{y}=201 \times 201 .
$$

\begin{tabular}{cccc}
\hline \multicolumn{4}{c}{$T^{*}$} \\
\hline$M_{\theta} \times M_{\varphi}$ & $y / H=0.2$ & $y / H=0.7$ & $y / H=0.9$ \\
$4 \times 4$ & 0.8266 & 0.6123 & 0.5374 \\
$5 \times 5$ & 0.8266 & 0.6124 & 0.5374 \\
$6 \times 6$ & 0.8235 & 0.6123 & 0.5374 \\
\hline
\end{tabular}




\section{Table 6}

Computational time to obtain a converged solution of $T^{*}$ for $H=3 h, H=(2+\sqrt{5}) h$, at $k_{a}=1 m^{-1}, N_{x} \times N_{y}=251 \times 251$.

\begin{tabular}{ccccc}
\hline & \multicolumn{5}{c}{ CPU time } \\
\hline \multirow{4}{*}{$H=3 h$} & $M_{\theta} \times M_{\varphi}$ & $N_{p l}=1$ & $N_{p l}=0.1$ & $N_{p l}=0.01$ \\
\cline { 2 - 5 } & $4 \times 4$ & $7 \min 32 \mathrm{~s}$ & $7 \min 34 \mathrm{~s}$ & $7 \min 35 \mathrm{~s}$ \\
& $5 \times 5$ & $10 \min 49 \mathrm{~s}$ & $10 \min 50 \mathrm{~s}$ & $10 \min 52 \mathrm{~s}$ \\
& $6 \times 6$ & $14 \min 52 \mathrm{~s}$ & $14 \min 54 \mathrm{~s}$ & $14 \min 56 \mathrm{~s}$ \\
$H=(2+\sqrt{5}) h$ & & & & \\
& $4 \times 4$ & $11 \min 56 \mathrm{~s}$ & $12 \min 1 \mathrm{~s}$ & $12 \min 2 \mathrm{~s}$ \\
& $6 \times 5$ & $15 \min 50 \mathrm{~s}$ & $15 \min 52 \mathrm{~s}$ & $15 \min 59 \mathrm{~s}$ \\
& $6 \times 6$ & $20 \min 11 \mathrm{~s}$ & $20 \min 15 \mathrm{~s}$ & $20 \min 16 \mathrm{~s}$ \\
\hline
\end{tabular}

Table 4 shows how the cell numbers modify quantitatively the values of $T^{*}$ in the medium. So, higher the cell numbers are, more the results are approximated accurately. The series expansions of $\operatorname{Bis}_{n}(u, \theta)$, with $u \in\left[0, k_{a} H\right]$ and $\left.\theta \in\right] 0, \frac{\pi}{2}[$ remain accurate up to the value of $u \leq 8$. Then, one should be careful on the choice of the number of terms in the series above this limit because of the round-off errors; why a double precision programming is recommended to evaluate the results expected [24]. Table 5 shows the effects of discrete directions in the computation of $T^{*}$ values. One can note that, when $y / H=0.9$ there is no a significant change on results when the discrete directions variate. This is because heat transfer by radiation, becomes weak when $y / H \geq$ 0.9 and $N_{p l}=0.1$. In contrast, there is a slight change on results when one reaches close to the hot boundary surface. Table 6 gives prominence to the fact that, as the dimensions of the square obstacle increase, the CPU time also increases. This is because the number of sub-domains where the integral solutions of the radiative transfer equations have been calculated and computed had increased. So, one can also note that the CPU time is greater in the case of complex geometry compared with the one of simple geometry $(h=0)$ and as $N_{p l}$ increases, the CPU time increases slightly for this complex geometry.

\subsubsection{Numerical simulation of conductive-radiative quantities}

- case: $H=3 h$ 

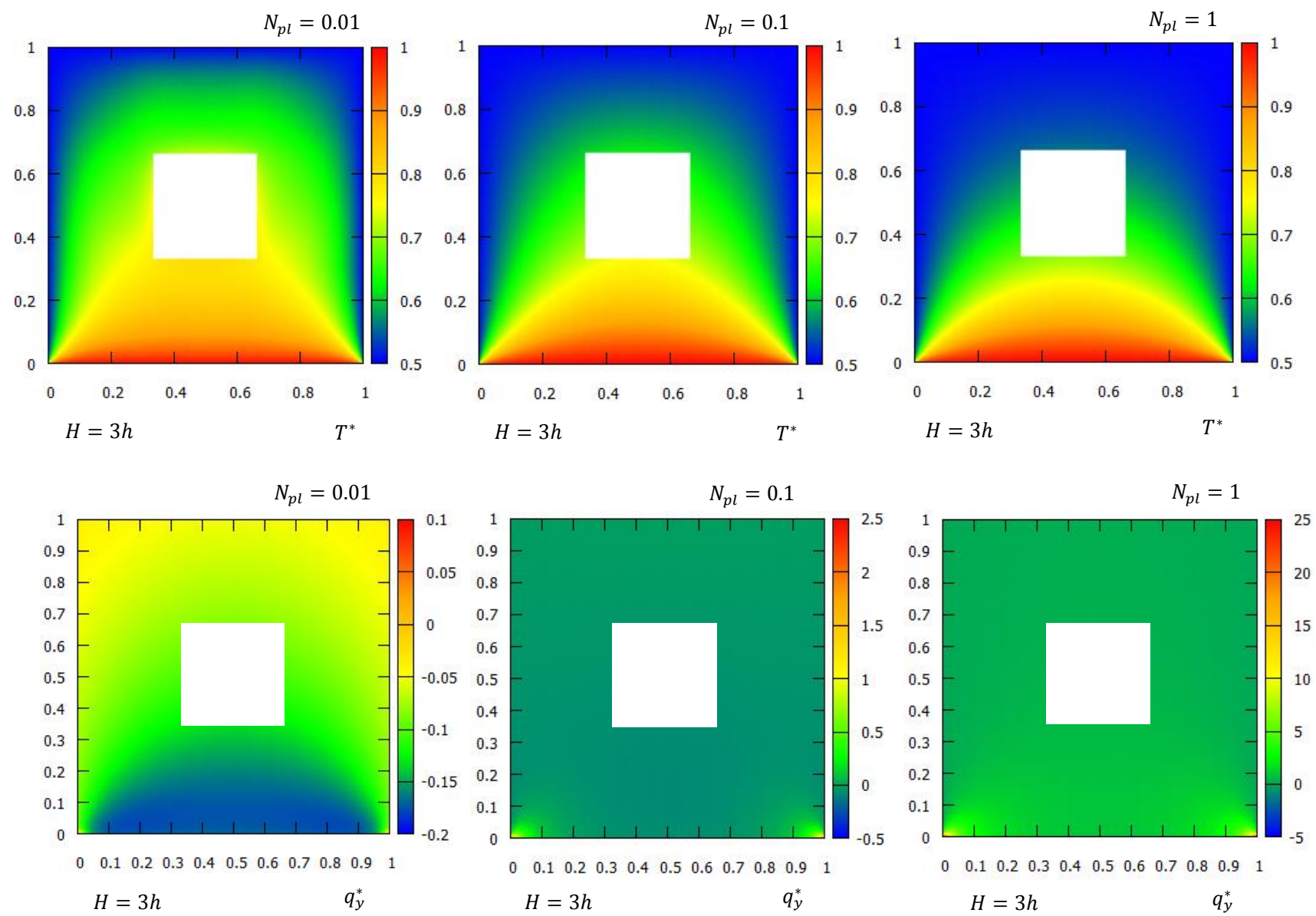

Fig.10. Dimensionless temperature distribution $T^{*}$ and total heat flux $q_{y}^{*}: H=3 h$, south boundary surface is hot, $k_{a}=1 m^{-1}, N_{x}=N_{y}=251, N_{p l} \in\{0.01,0.1,1\}$.

Fig. 10 illustrates the effect of conduction-radiation parameter on the dimensionless temperature and the total heat flux fields, when $H=3 h, k_{a}=1 \mathrm{~m}^{-1}$ and $N_{x}=N_{y}=251$. The results of $T^{*}$ presented inform that, more $N_{p l}$ increases, less heat transfer propagates in the participating medium. Similar simulations have been shown in the case of radiation heat transfer [23], but with different physical profiles. When $N_{p l}=0.01$, there are more hot points in the participating medium, but those hot points decrease when $N_{p l}$ increases because of the medium that strongly absorbs radiations. Thus, similar observations are made at Fig. 4 in the sub-section 3.1.1, except the fact; there is an obstacle in this case. The results of $q_{y}^{*}$ show that, when $N_{p l}=0.01$, heat transfer in the medium is ensured by radiation. This analysis remains in accordance with Eq. (34), and the profile is very close to Fig. 5 obtained when there is no obstacle, but with different data because some rays have been blocked. When $N_{p l}$ reaches the unit value, conduction heat transfer takes over, mostly the hot south boundary surface contributes to energy transfer, and the wall heat fluxes can be deduced.

- Case : $H=(2+\sqrt{5}) h$ 


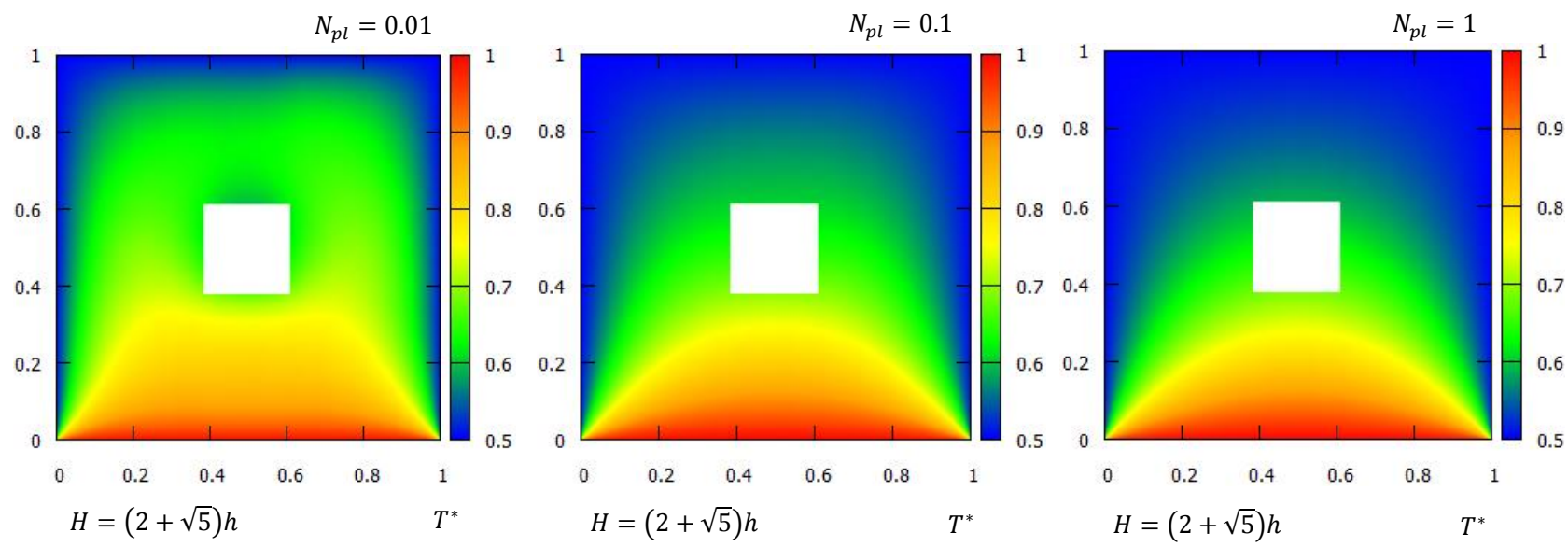

Fig.11. Dimensionless temperature distribution $T^{*}: H=(2+\sqrt{5}) h$, south boundary surface is hot,

$$
k_{a}=1 m^{-1}, N_{x}=N_{y}=251, N_{p l} \in\{0.01,0.1,1\} .
$$

When the size of the centered obstacle decreases on Fig. 11, the number of hot points in the medium increases. Since conduction heat transfer is looked as a local phenomenon, when $N_{p l}=$ 1 , the obstacle doesn't modify temperature profile because the effect of radiation becomes not effective. Whereas, at $N_{p l}=0.01$, the blockage of radiations from hot boundary surface modifies the curve shape of $T^{*}$, but keep the symmetry because of the geometry. When $N_{p l}=0.1$, it is one of the best cases to analyze the combined conduction-radiation phenomenon, because neither conduction, nor radiation predominates.

- Case : $H=8 h$

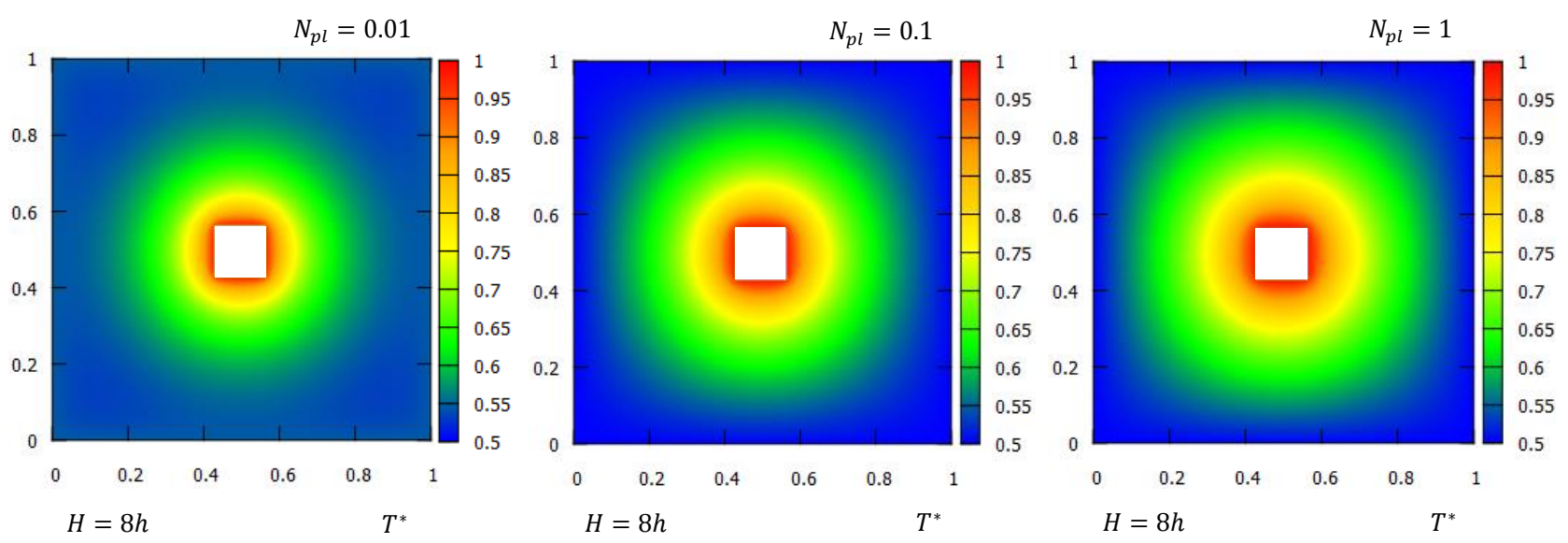

Fig.12. Dimensionless temperature distribution $T^{*}: H=8 h$, external boundary surfaces of the centered obstacle are hot, $k_{a}=1 \mathrm{~m}^{-1}, N_{x}=N_{y}=251, N_{p l} \in\{0.01,0.1,1\}$. 
Numerical simulations shown at Fig. 12 have been performed when the external surfaces of the centered obstacle are kept hot $T_{r e f}$, but the boundary surfaces of the 2D enclosure are maintained cold, at the initial temperature in the medium $T_{0}$. Absorption coefficient is $k_{a}=1 \mathrm{~m}^{-1}$, corresponding to a unit optical thickness $\left(\tau=k_{a} H\right)$, with $H=1 \mathrm{~m}$. In this case, the size of the centered obstacle is small and for $N_{p l}=0.1$, neither conduction nor radiation predominates one on the other. The curves describing the set of hot points in the participating medium follow concave profiles around the centered obstacle. The number of the hot points concerned variates with the conduction-radiation parameter. Therefore, more $N_{p l}$ increases, better the number of hot points is. When $N_{p l}$ increases and reaches a high value, the number of hot points also increases and reaches a threshold; because only conduction predominates at this moment in the process of heat transfer.

With regard to the suitable previous simulations, the complexity of the geometry makes increase the number of sub-domains where the semi-analytical solutions are calculated. Therefore, each given geometry corresponds to different kind of equations modelling the problem and the smaller the dimensions of the obstacle, the greater the difficulty on simulations. By the way, as compared with the numerical methods like DOM, the present one doesn't suffer from ray effect when one solves the radiative transfer equation [23] before combining it to the energy equation.

\subsubsection{Effect of the obstacle on conduction-radiation temperature}

The aim of this subsection is to present how the obstacle modifies quantitatively heat transfer in the participating medium. Therefore, Table.5 illustrates it with the corresponding values of $T^{*}$ for $h=\{0, \sqrt{5}-2,1 / 3\} H$ along the y-direction of the $2 \mathrm{D}$ enclosure. Thus, the choice of $N_{p l}=0.1$ is made because there is a good interaction between conduction and radiation heat transfer. So, there is a slight difference on the values of $T^{*}$ obtained when $h=0$, compared with those of $h \neq 0$, because of blockage of rays in the medium. When, $h=(\sqrt{5}-2) H$, and $h=H / 3$, the radiation contributes less because the size of the obstacle becomes more important, then some rays from top, bottom, left and right sides of the participating medium are blocked. Consequently, more the size of the obstacle decreases more the values of $T^{*}$ converges to those computed with $h=0$. If one continues this investigation for low value of $N_{p l}$, some results already established for pure radiation [23] can be used. On the other hand, if $N_{p l}$ takes large values, radiation heat transfer becomes ineffective and the obstacle does not affect heat transfer, because conduction heat transfer is a local phenomenon.

Table.7

Effect of the obstacle on conductive-radiative dimensionless temperature, $N_{p l}=0.1, k_{a}=1 \mathrm{~m}^{-1}, N_{x}=N_{y}=251$ at the centerline position $x / H=0.5$. 


\begin{tabular}{cccc}
\hline \multicolumn{3}{c}{$T^{*}$} \\
\hline$y / H$ & $h=0$ & $h=(\sqrt{5}-2) H$ & $h=(1 / 3) H$ \\
\hline 0.0 & 1.000 & 1.000 & 1.000 \\
0.1 & 0.896 & 0.895 & 0.894 \\
0.2 & 0.818 & 0.814 & 0.812 \\
0.3 & 0.755 & 0.745 & 0.744 \\
0.4 & 0.703 & $\ldots$ & $\ldots$ \\
0.5 & 0.661 & $\ldots$ & $\ldots$ \\
0.6 & 0.624 & $\ldots$ & $\ldots .582$ \\
0.7 & 0.591 & 0.584 & 0.550 \\
0.8 & 0.560 & 0.550 & 0.530 \\
0.9 & 0.530 & 0.530 & 0.500 \\
1.0 & 0.500 & 0.500 & \\
\hline
\end{tabular}

\subsubsection{Transient state}
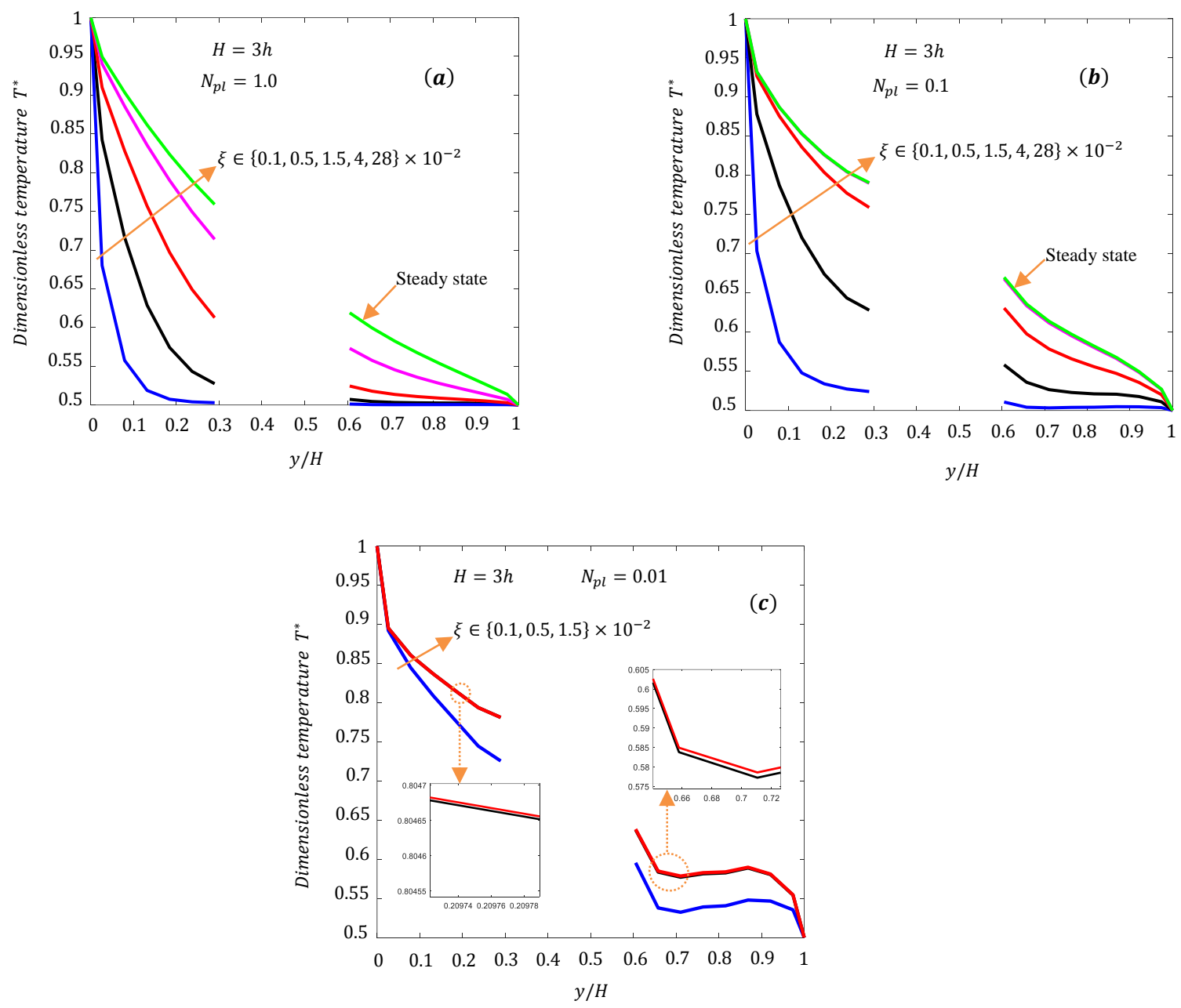
Fig.13. Transient dimensionless temperature $T^{*}$ in the participating medium when $H=3 h, k_{a}=1 \mathrm{~m}^{-1}$ at different instant $\xi$,with $N_{p l} \in\{0.01,0.1,1\}$ for (a), (b) and (c) respectively.

The numerical results of $T^{*}$ at different instant $\xi$ and with different values of $N_{p l}$ along a centerline position $x / H=0.5$ are presented in the case of $H=3 h, \Delta \xi=5.10^{-4}, N_{x}=N_{y}=21$. The numerical simulations of dimensionless temperature distribution at different instant $\xi$ are also displayed and discussed when $H=3 h$ and $H=(2+\sqrt{5}) h$.

When $N_{p l} \in\{0.01,0.1,1\}$, one can observe through the shape of the curves shown in Fig. 13 that one gets closer to the steady state as the time increases. Unfortunately, the steady state is not reached there during the same elapsed time, because the more $N_{p l}$ decreases, the faster it is reached. In this case dealing with complex geometry, the obstacle blocks rays coming from north, south, est and west sides of the 2-D enclosure. When the value of $N_{p l}$ decreases and facilitates the predominance of radiation, all the rays will not fully participate in the heat transfer because of the obstacle which will block them. Consequently, in the same conditions when the values of $N_{p l}$ decrease, the obstacle modifies quantitatively and qualitatively the field of conduction-radiation temperature in the semi-transparent medium.

\subsubsection{Numerical simulations}

The numerical simulations presented concern the geometries $H=3 h$, and $H=(2+\sqrt{5}) h$. The dimensionless step time $\Delta \xi=10^{-6}$ and $N_{x}=N_{y}=251$ have been used to respect the condition of stability and to display suitable simulations.

- Case: $H=3 h$

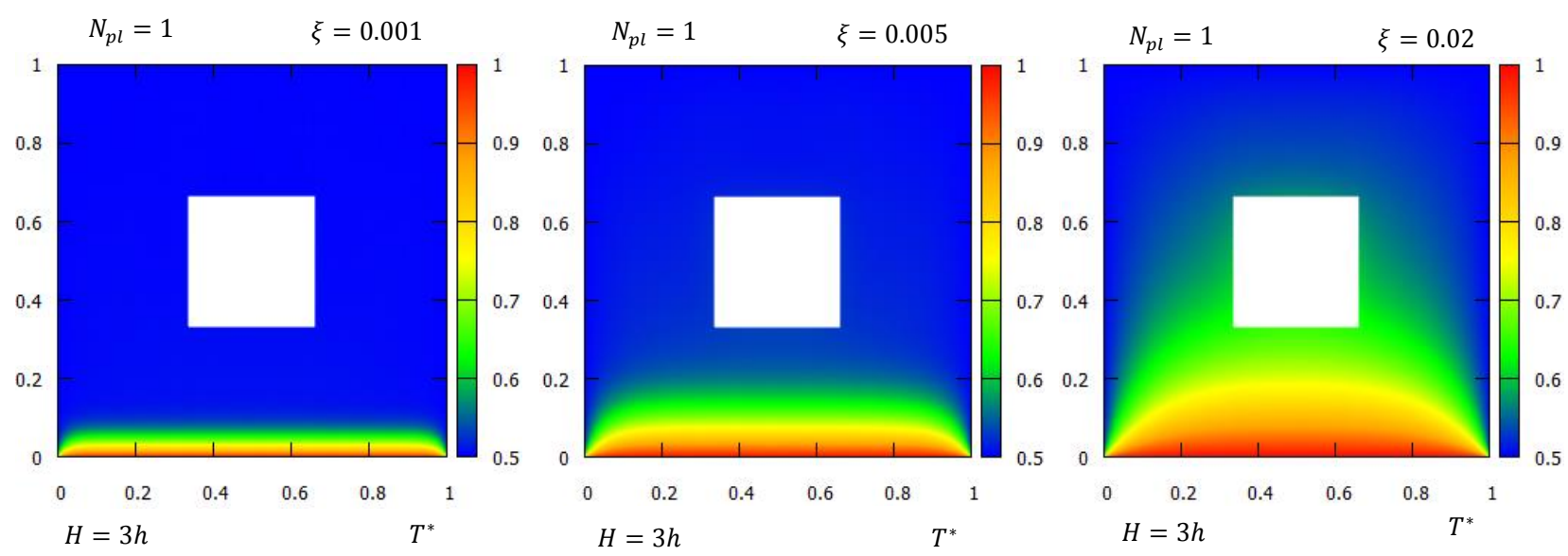

Fig. 14. Transient dimensionless temperature distribution $T^{*}$ for $H=3 h$, south boundary surface is hot, $k_{a}=1 m^{-1}, N_{x}=N_{y}=251, N_{p l}=1, \xi=\{1,5,20\} \times 10^{-3}$. 


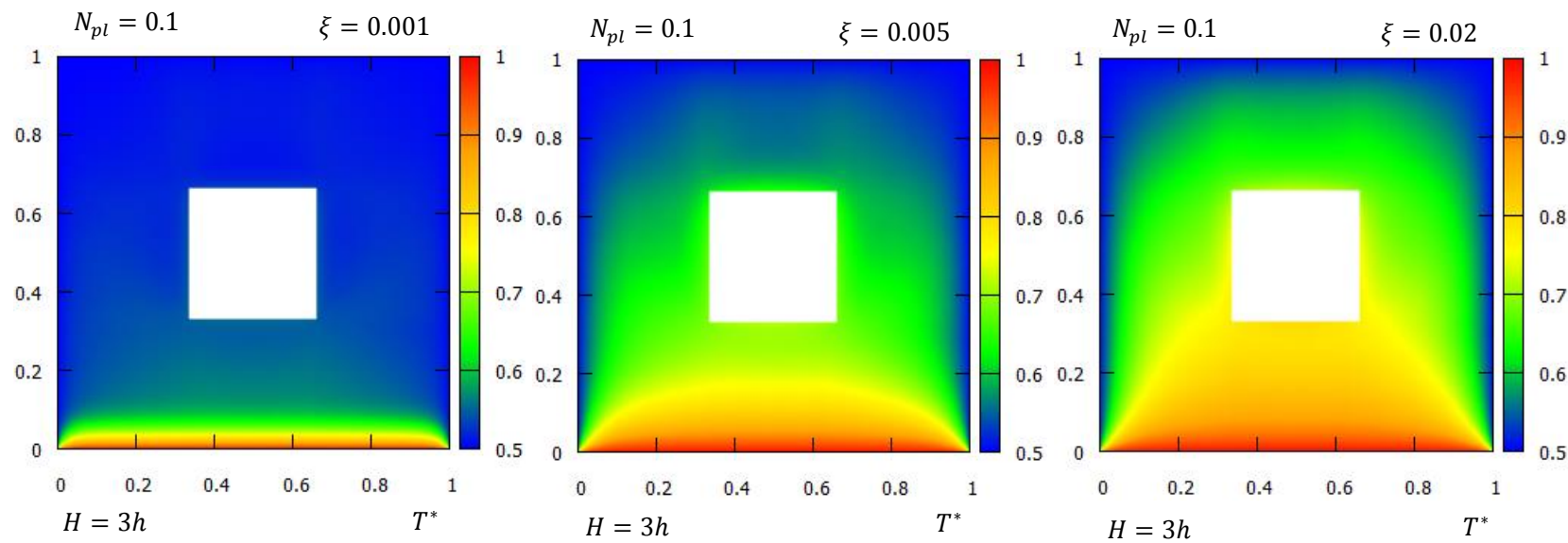

Fig. 15. Transient dimensionless temperature distribution $T^{*}$ for $H=3 h$, south boundary surface is hot, $k_{a}=1 m^{-1}, N_{x}=N_{y}=251, N_{p l}=0.1, \xi=\{1,5,20\} \times 10^{-3}$.

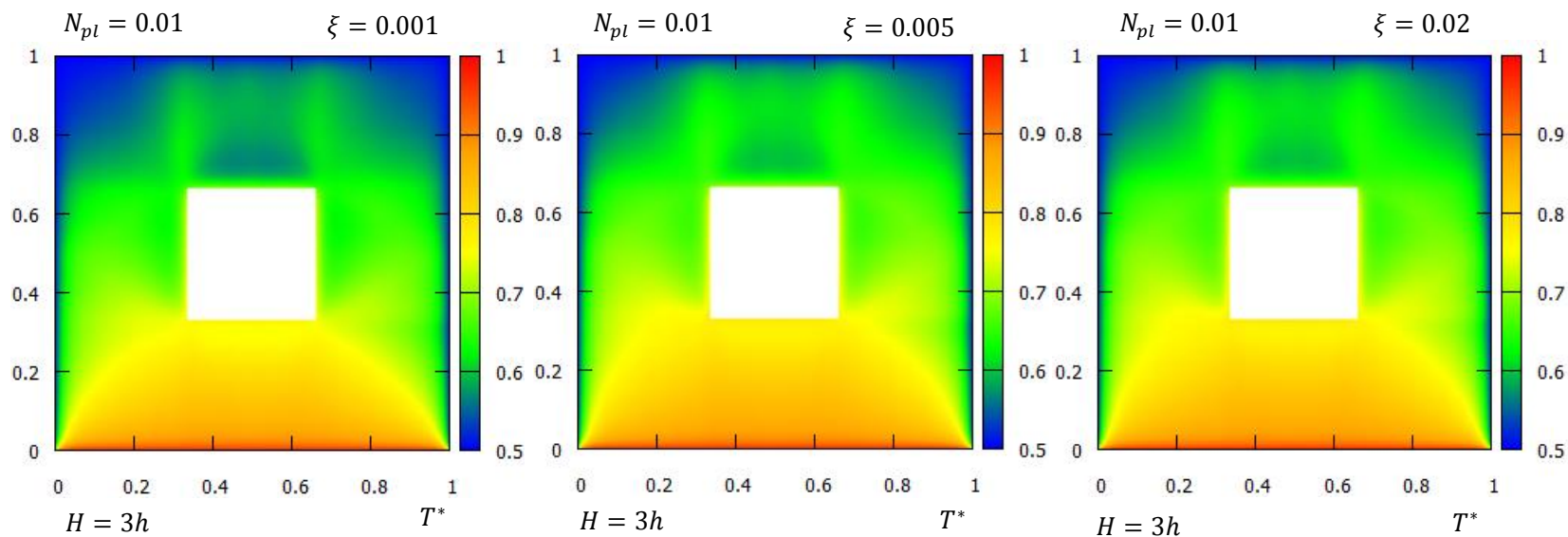

Fig. 16. Transient dimensionless temperature distribution $T^{*}$ for $H=3 h$, south boundary surface is hot, $k_{a}=1 m^{-1}, N_{x}=N_{y}=251, N_{p l}=0.01, \xi=\{1,5,20\} \times 10^{-3}$.

When $N_{p l}=1$ and $\xi=\{1,5,20\} \times 10^{-3}$, heat spreads slowly from the south boundary to the north one of the participating medium but keeps it symmetry with respect to the line $y=0.5$. Steady state takes considerable time to reach when $N_{p l}=1$, because of conduction predominance. When $N_{p l}=0.1$ there is a faster propagation of heat between $0.001<\xi \leq 0.005$ compared with the case of $N_{p l}=1$. This is because radiation spreads fastly there during a short time; furthermore, when $\xi>0.02$ one tends towards steady state. When $N_{p l}=0.01$, radiation imposes that heat propagates very quickly by deflecting the obstacle until reaching the north boundary in a very short time. Consequently, at $\xi=0.005$ steady state is already reached compared with those cases where $N_{p l}$ takes the values of 1 and 0.1 . These proposed numerical simulations also approve the behavior of the 1D numerical results presented in Fig. 13. 
- Case: $H=(2+\sqrt{5}) h$

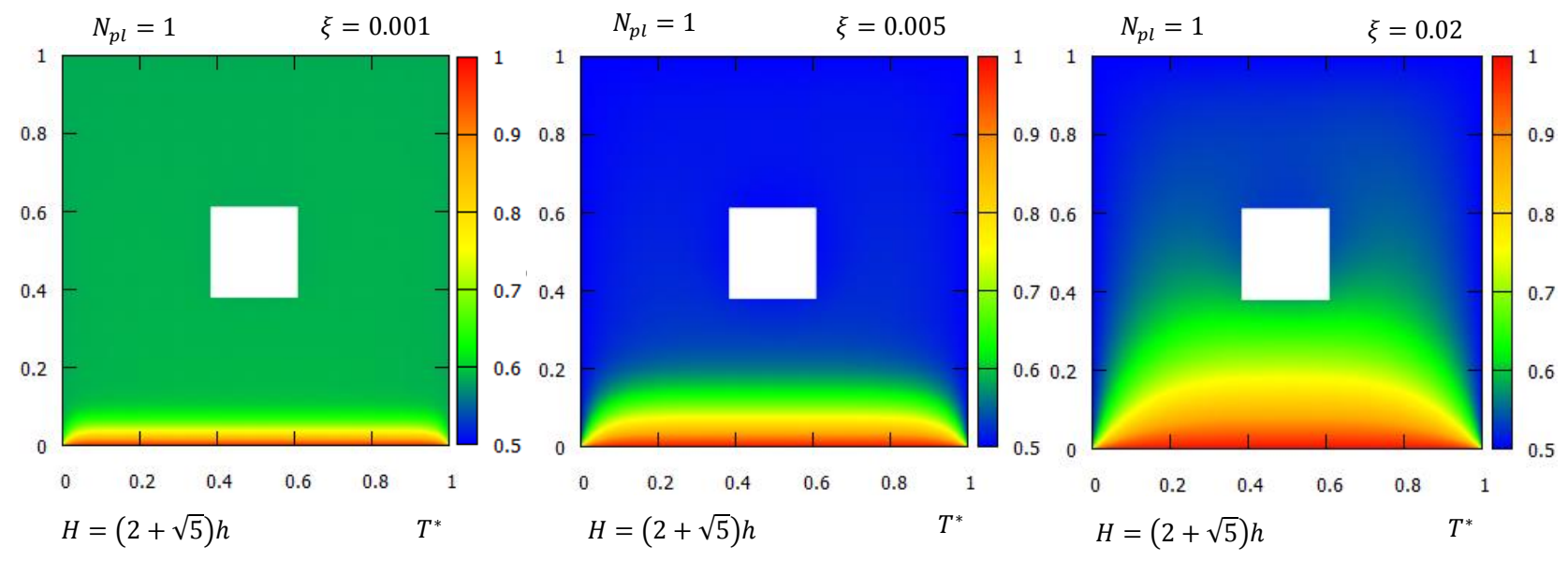

Fig. 17. Transient dimensionless temperature distribution $T^{*}$ for $H=(2+\sqrt{5}) h$, south boundary surface is hot, $k_{a}=1 m^{-1}, N_{x}=N_{y}=251, N_{p l}=1, \xi=\{1,5,20\} \times 10^{-3}$.

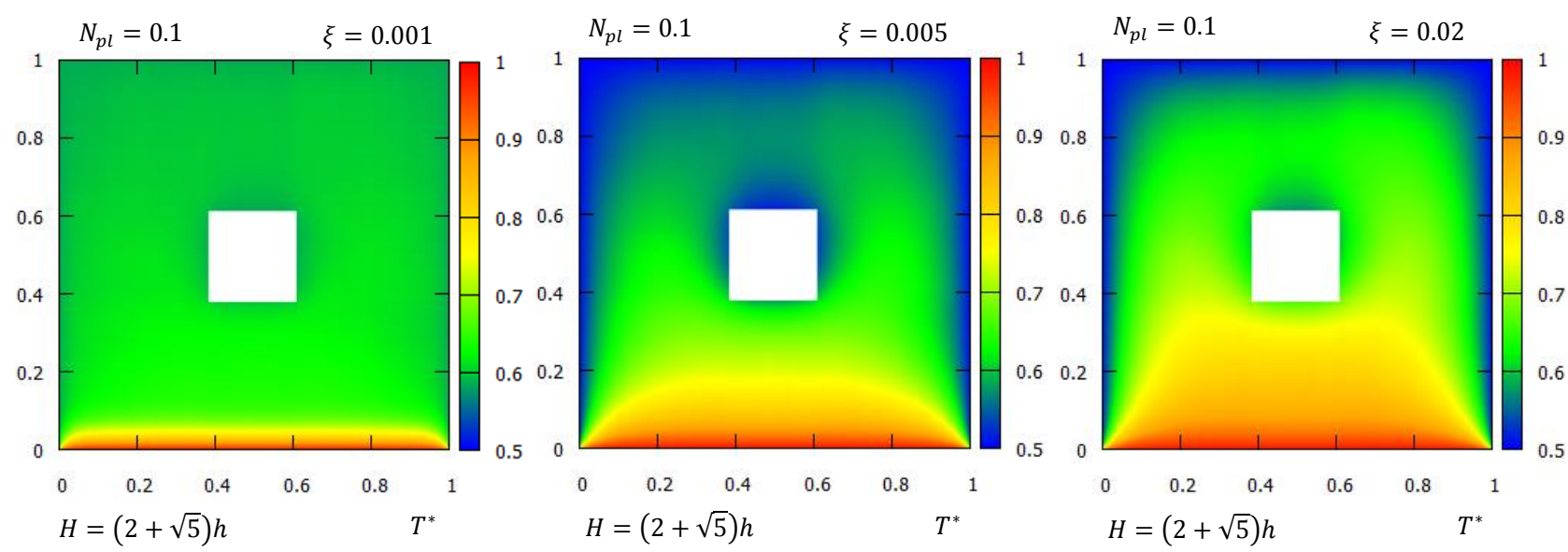

Fig. 18. Transient dimensionless temperature distribution $T^{*}$ for $H=(2+\sqrt{5}) h$, south boundary surface is hot, $k_{a}=1 m^{-1}, N_{x}=N_{y}=251, N_{p l}=0.1, \xi=\{1,5,20\} \times 10^{-3}$.

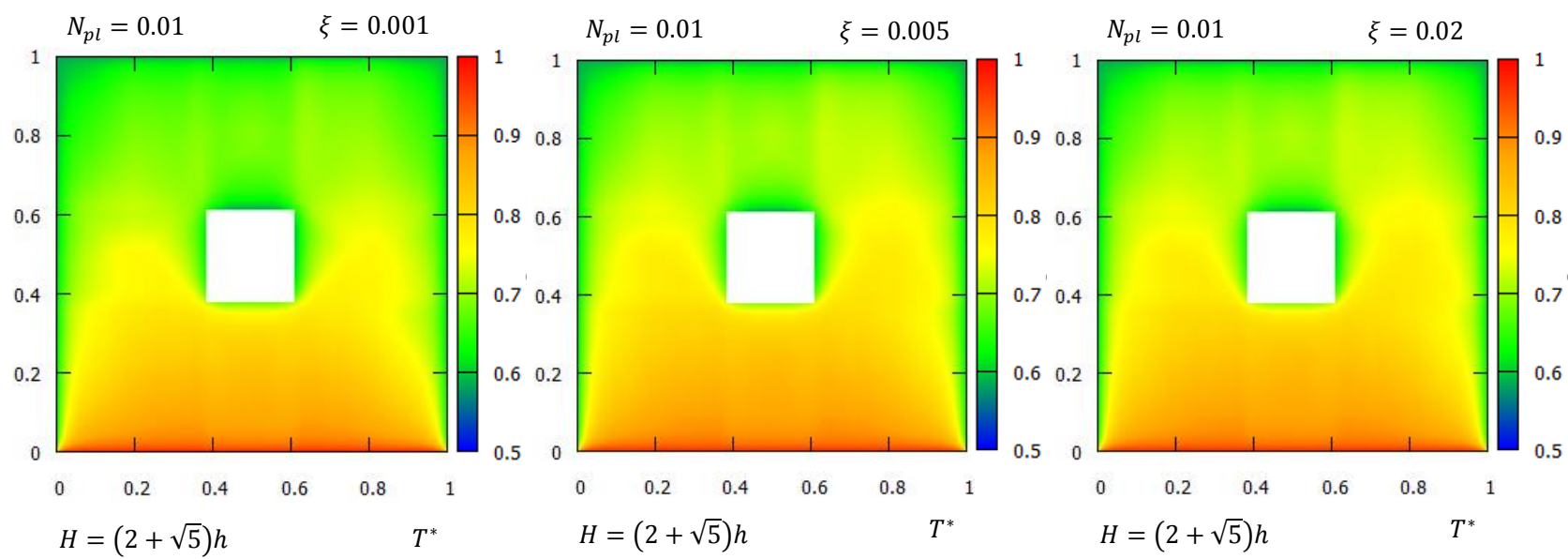


Fig. 19. Transient dimensionless temperature distribution $T^{*}$ for $H=(2+\sqrt{5}) h$, south boundary surface is hot, $k_{a}=1 m^{-1}, N_{x}=N_{y}=251, N_{p l}=0.01, \xi=\{1,5,20\} \times 10^{-3}$.

Fig. 17, Fig. 18, and Fig. 19 above illustrate the numerical simulations of $T^{*}$ with the use of parameters $N_{p l} \in\{001,0.1,1\}$ at different instant $\xi \in\{1,5,20\} \times 10^{-3}$ and when the size of the obstacle obeys to $H=(2+\sqrt{5}) h$. The behavior of the simulations here is not far from those presented on Fig. 16, but the obstacle is smaller and enables more rays travel from south to north boundaries. There is a slight problem of symmetry there because it becomes difficult to mesh the geometry when the size of the obstacle is small.

With regard to the deep analysis carried out, although the present method uses the exact calculation of the integral form of radiative transfer equation, it provides suitable results for optically thick medium [23]. Nevertheless, for the combined conductive-radiative heat transfer, when $N_{p l}$ becomes close to zero with an optical thickness $\tau \geq 5$ the present method leads to slight errors in computations.

\section{Conclusion}

An investigation on the combined conduction-radiation heat transfer in 2D semi-transparent medium with a centered obstacle has been carried out. Exact semi-analytical and then, numerical formulation of the problem has been presented. Modelling of radiation heat transfer has been established similarly with a ray tracing method adapted for the present geometry, and energy equation have been computed with the centered finite differences method. Conduction-radiation temperature and the total heat flux have been calculated, simulated then displayed both for simple and complex geometries. The effect of the conduction radiation parameter, the cell numbers, the discrete directions and the size of the cavity have been discussed. Numerical results of dimensionless temperature when there is no obstacle in the medium have been compared with the literature in good accuracy, both at steady and transient states. Several simulations of the semitransparent medium have also been proposed when there is variation of the conduction-radiation parameter and the size of the obstacle. The present method used to solve the conductive-radiative problem in complex geometry, have been realized with satisfactory computation time and without ray effect from radiation. Without some difficulties encountered with the use of regular grids in this irregular geometry, the method is promising to solve the conductive-radiative heat transfer in complex geometries. Later, one intends to combine convection-conduction and radiation of real gases or fluid flow filled in a similar geometry with diffusely reflecting boundaries.

\section{Acknowledgment}

Institut Pprime, University of Poitiers, CNRS and ENSMA UPR 3346 in France full supported this work. 


\section{Appendix A. Dimensionless parameters}

Time: $\xi=\frac{D}{H^{2}} t$, where $D$ is the thermal diffusivity given by $D=k_{c} / \rho C_{p}$.

Space coordinates: $\quad x^{*}=\frac{x}{H} ; \quad y^{*}=\frac{y}{H}$.

Temperatures: $\quad T^{*}=\frac{T}{T_{r e f}} ; \quad T_{0}^{*}=\frac{T_{0}}{T_{r e f}}$.

Radiative quantities: $\quad G^{*}=\frac{G}{4 \sigma_{B} T_{r e f}^{4}} ; \quad q^{r^{*}}=\frac{q^{r}}{4 \sigma_{B} T_{r e f}^{4}}$ and $S^{r^{*}}=\frac{S^{r}}{4 \sigma_{B} T_{r e f}^{4}}$.

Total heat flux: $\quad q_{x}^{*}=\frac{q_{x}}{4 \sigma_{B} T_{r e f}^{4}} ; \quad q_{y}^{*}=\frac{q_{y}}{4 \sigma_{B} T_{r e f}^{4}}$.

Planck number: $\quad N_{p l}=\frac{k_{c} k_{a}}{4 \sigma_{B} T_{r e f}^{3}}$.

\section{References}

[1] W.W. Yuen, E.E. Takara, Analysis of combined conductive-radiative heat transfer in a two dimensional rectangular enclosure with a gray medium, ASME J. Heat Transfer 110 (1988) 468-474.

[2] T.Y. Kim, S.W. Baek, Analysis of combined conductive and radiative heat transfer in a two dimensional rectangular enclosure using the discrete ordinates method, Int. J. Heat Mass Transfer 34 (1991) 2265-73.

[3] K. H. Lee, R. Viskanta, Two-dimensional combined conduction and radiation heat transfer: comparison of the discrete ordinates method and the diffusion approximation methods, Numer. Heat Transfer A 39 (2001) 205-25.

[4] W.W. Yuen, L.W. Wong, Analysis of radiative equilibrium in a rectangular enclosure with gray medium, ASME J. Heat Transfer 106 (1984) 433-440.

[5] W.W. Yuen, Analysis of radiative equilibrium in a rectangular enclosure with gray medium: cases with diffusely reflecting surfaces and heat flux boundary conditions, ASME Winter Annual Meeting (1984), New Orléans.

[6] S.C. Mishra, A. Lankadasu, K.N. Beronov, Application of the lattice Boltzmann method for solving the energy equation of a 2-D transient conduction-radiation problem, Int. J. Heat Mass Transfer 48 (2005) 3648-3659.

[7] M. Sakami, A. Charette, V. Le Dez, Application of the discrete ordinates method to combined conductive and radiative heat transfer in two-dimensional complex-geometry, J. Quant. Spectrosc. Radiat. Transf. 56 (1996) 517-533.

[8] D.R. Rousse, Numerical predictions of two dimensional conduction, convection and radiation heat transfer-I. Formulation. Int. J. Therm. Sci. 39 (2000) 315-331. 
[9] S.K. Mahapatra, B.K. Dandapat, Analysis of combined conduction and radiation heat transfer in presence of participating medium by development of hybrid method, J. Quant. Spectrosc. Radiat. Transf. 102 (2006) 277-292.

[10] J.F. Luo, X. Shen, Numerical method of the ray tracing-node analyzing method for solving 2-D transient coupled heat transfer in a rectangular semi-transparent medium, Numer. Heat Transf. A 55 (2009) 465-486.

[11] M. Lazard, Heat transfer in a semi-transparent parallelogram shaped medium, IJHT 34 (2) (2016) S420-S424.

[12] M. Ghattassi, J.R. Roche, F. Asllanaj, M. Boutayeb, Galerkin method for solving combined radiative and conductive heat transfer, Int. J. Therm. Sci. 102 (2016) 122-136.

[13] M.M. Keshtkar, P.T. Talebizadehsardari, Investigation of transient conduction-radiation heat transfer in a square cavity using combinaison of LBM and FVM, Sädhanā 43 (2018) 64.

[14] F. Asllanaj, G. Parent, G. Jeandel, Transient Radiation and conduction heat transfer in a gray absorbing-emitting medium applied on two-dimensional complex-shaped domains, Numer. Heat Transf. B 52 (2) (2007) 179-200.

[15] H. Amiri, S.H. Mansouri, A. Safavinejad, Combined conductive and radiative heat transfer in an anisotropic scattering participating medium with irregular geometries, Int. J. Therm. Sci. 49 (2010) 492-503.

[16] K. Lari, S.A. Gandjalikhan Nassab, Analysis of combined radiative and conductive heat transfer in three-dimensional complex geometries using blocked-off method, Trans. Mech. Eng. 35 (2011) 107-119.

[17] P. Talukdar, M.A.A. Mendes, R. K. Parida, D. Trimis, S. Ray, Modelling of conductionradiation in porous medium with blocked-off region approach, Int. J. Therm. Sci. 72 (2013) 102-114.

[18] M. Zare, S. A. Gandjalikhan Nassab, combined radiative-conductive heat transfer in twodimensional complex geometries with variable thermal conductivity, T. Mech. Eng. 38 (2014) 275-288.

[19] M.M. Keshtkar, B. Amiri, Numerical simulation of radiative-conductive heat transfer in an enclosure with an isotherm obstacle, Heat Transf. Eng. 39 (2018) 72-83.

[20] M.M. Addini, S. A. Gandjalikhan Nassab, Combined mixed convection and radiation heat transfer in an obstacle wall mounted lid-driven cavity, Int. J. Nonlin. Sci. Num. 17 (6) (2016).

[21] S. Saravanan, C. Sivaraj, Coupled thermal radiation and natural convection heat transfer in a cavity with a heated plate inside, Int. J. Heat Fluid Fl. 40 (2013) 54-64.

[22] J.S. Djeumegni, M. Lazard, V. Le dez, H.T.T. Kamdem, Modeling of radiative heat transfer in a gray semi-transparent medium with internal fluid cavity limited by black boundary surfaces, TI-Int. J. Eng. Sci 63 (2019) 205-210.

[23] J.S. Djeumegni, M. Lazard, V. Le dez, H.T.T. Kamdem, Radiative heat transfer in a 2D semitransparent gray medium with a centered inner square cavity, Int. J. Heat Mass transfer 149 (2020) 119209.

[24] Z. Altaç, Integrals involving Bickley and Bessel function in radiative transfer and generalized exponential integral function, J. Heat Transfer 118 (3) (1996) 789-791. 
[25] K.A.R. Ismail, C.T.S. Salinas, gray radiative conductive 2D modeling using discrete ordinates method with multidimensional spatial scheme and non-uniform grid, Int. J. Therm. Sci. 45 (7) (2006) 706-715.

[26] S. Wang, R. Ni, Solving of two-dimensional unsteady-state heat-transfer inverse problem using finite difference method and model prediction control method, Hindawi, (2019) 1-12.

[27] M. Sakami, A. Charette, V. Le Dez, Application of the discrete ordinates method to combined conductive and radiative heat transfer in a two-dimensional complex geometry, J. Quant. Spectrosc. Radiat. Transfer, 56 (1996) 517-633.

[28] D.R. Rousse, G. Gautier, J.F. Sacadura, Numerical Predictions of two-dimensional conduction, convection and radiation heat transfer. II. Validation, Int. J. Thermal Sci., 39 (2000) 332-353.

[29] P. Talukdar, F.V. Issendorff, D. Trimis, and C.J. Simonson. Conduction-radiation interaction in 3d irregular enclosures using the finite volume method, Heat Mass Transfer, 44(6):695-704, 2008.

[30] C.Y. Wu, N.R. Ou, Transient two-dimensional radiative and conductive heat transfer in a scattering medium, Int. J. Heat Mass Transfer 37 (1994) 2675-2686.

[31] S.C. Mishra, P. Talukdar, D. Trimis, F. Durst, computational efficiency improvements of the radiative transfer problems with or without conduction-a comparison of the collapsed dimension method and the discrete transfer method, Int. J. Heat Mass Transf. 46 (2003) 30833095.

[32] S.C. Mishra, H.K. Roy, solving transient conduction and radiation heat transfer problems using the lattice Boltzmann method and the finite volume method, J. Comput. Phys. 223 (2007) 89-107.

[33] S.C. Mishra, M.Y. Kim, S. Maruyama, Performance evaluation of four radiative transfer methods in solving multi-dimensional radiation and/or conduction heat transfer problems, Int. J. Heat Mass Transf. 55 (2012) 5819-5835. 